\title{
Optimal investment with inside information and parameter uncertainty
}

\author{
Albina Danilova \\ Department of Mathematics \\ London School of Economics and Political Science \\ ANDREW NG \\ Mathematical Institute \\ University of Oxford
}

Michael Monoyios*

Mathematical Institute

University of Oxford

November 6, 2009

\begin{abstract}
An optimal investment problem is solved for an insider who has access to noisy information related to a future stock price, but who does not know the stock price drift. The drift is filtered from a combination of price observations and the privileged information, fusing a partial information scenario with enlargement of filtration techniques. We apply a variant of the Kalman-Bucy filter to infer a signal, given a combination of an observation process and some additional information. This converts the combined partial and inside information model to a full information model, and the associated investment problem for HARA utility is explicitly solved via duality methods. We consider the cases in which the agent has information on the terminal value of the Brownian motion driving the stock, and on the terminal stock price itself. Comparisons are drawn with the classical partial information case without insider knowledge. The parameter uncertainty results in stock price inside information being more valuable than Brownian information, and perfect knowledge of the future stock price leads to infinite additional utility. This is in contrast to the conventional case in which the stock drift is assumed known, in which perfect information of any kind leads to unbounded additional utility, since stock price information is then indistinguishable from Brownian information.
\end{abstract}

\section{Introduction}

The goal of this paper is to examine the combined influence of inside information and drift parameter uncertainty on optimal investment rules. To this end, we explicitly solve a one-dimensional Merton-style investment problem for an insider who possesses, at time zero, additional information beyond that of regular traders, but who is not assumed to know the value of the stock's appreciation rate. Although the assumption of a one-dimensional model with unknown constant drift is restrictive, it has the benefit of allowing us to derive fully explicit solutions, so that the effect of the inside information on the parameter estimation and the optimal investment rule can be fully gauged.

This work thus combines elements of partial information models such as those of Rogers [28] or Björk, Davis and Landén [7], with enlargement of filtration techniques to incorporate the insider's additional information, as pioneered by Pikovsky and Karatzas [27].

The insider has knowledge of the value of a random variable $L$, corresponding to (usually, noisy) knowledge of the terminal stock price or of the terminal value of the Brownian motion driving the stock. But the insider does not have access to the Brownian filtration. Her trading strategies are required to be adapted to the stock price filtration, but enlarged by the additional

\footnotetext{
* corresponding author
} 
information. The stock's risk premium is then an unobservable signal process which is estimated via a Kalman-Bucy filter. The filtering algorithm, Proposition 3, computes the best estimate of the stock's risk premium given both stock price observations and the additional information. In this case the usual Kalman-Bucy equations hold, but with modified initial conditions reflecting the additional information.

We begin with lognormal stock price dynamics written under some background filtration $\mathbb{F}$, under which the stock drift is known. We enlarge $\mathbb{F}$ by $\sigma(L)$, using classical techniques of initial enlargement of filtration $[18,24,30]$. Denoting the enlarged filtration by $\mathbb{F}^{L}$, the effect of the enlargement is to decompose an $\mathbb{F}$-Brownian motion into an $\mathbb{F}^{L}$-Brownian motion plus an information drift, $\nu^{L}$, an $\mathbb{F}^{L}$-adapted process. The risk premium then becomes an $\mathbb{F}^{L}$-adapted process $\lambda^{L}$. With the dynamics of the stock price and its risk premium written under the enlarged filtration $\mathbb{F}^{L}$, the filtering algorithm infers the insider's unknown risk premium based on stock price observations as well as the additional information $\sigma(L)$.

If we denote by $\widehat{\mathbb{F}}^{L}$ the stock price filtration enlarged by $\sigma(L)$, the effect of the filtering is to convert the partial and inside information model to a standard full information model with random drift that is adapted to $\widehat{\mathbb{F}}^{L}$. Having restored a full information scenario, we solve the insider's utility maximisation problem using duality methods, giving closed form expressions for the maximum utility and optimal trading strategy.

We compare the results with the corresponding quantities for a regular agent who does not have inside information, but who must still filter the stock price drift. We find that stock price inside information is more valuable than information on the Brownian motion driving the stock. This can be traced directly to the parameter uncertainty, which requires stock price observations in order to be resolved. Hence, we find that exact terminal stock price information can lead to unbounded additional utility for the insider, but exact knowledge of the terminal Brownian motion does not. This is to be contrasted with the seminal insider trading model of Pikovsky and Karatzas [27]. In [27] there was no parameter uncertainty, so exact information of any kind led to unbounded logarithmic utility, as advance Brownian knowledge is equivalent to stock price knowledge when the stock's drift is known.

There are many papers on partial information investment models $[7,8,22,26,28,29]$, in which trading strategies are required to be adapted to the stock price filtration. There is also a rich literature on insider trading models, dating back to the classical equilibrium models of Kyle [21] and Back [5], built upon by Cho [11], Campi and Çetin [10], Danilova [13], and Aase, Bjuland and Øksendal [1]. In these models, the insider can use his additional information to influence the stock price, a feature that also appears in Kohatsu-Higa and Sulem [20].

Insider trading models in which techniques of enlargement of filtration play a direct role stem from Pikovsky and Karatzas [27]. Amendinger, Imkeller and Schweizer [3] gave an entropic characterisation of the additional utility achievable by an insider, extended to a semimartingale setting by Ankirchner, Dereich and Imkeller [4]. Amendinger, Becherer and Schweizer [2] used indifference arguments to give a monetary value to inside information in portfolio optimisation. Imkeller $[16,17]$ used the notion of progressive enlargement of filtration to model inside information on a random time that is not a stopping time for regular traders, and used Malliavin calculus to characterise the information drift. Corcuera et al [12] considered a dynamic flow of inside information, Baudoin and Nguyen-Ngoc [6] considered so-called weak information, involving knowledge of the law of some random variable, Hillairet [15] compared optimal strategies of insiders with different forms of side-information, and Campi [9] treated a quadratic hedging problem. To the best of our knowledge this paper is the first to combine partial and inside information scenarios.

The rest of the paper is organised as follows. In Section 2 we solve an investment problem involving a stock with a Gaussian drift process. We need this result as the subsequent utility maximisation problems can be rendered into this form. Section 3 details the model and the investment problems, and outlines our methodology. Section 4 gives the solution to the partial information investment problem with no inside information, which we use for comparison with later results. The main results, the solutions of the investment problems with inside information and parameter uncertainty, are given in Section 5. Section 6 gives the enlargement of filtration and filtering results that are applied in Section 7, where the main theorems are proven. Section 
8 concludes.

\section{Optimal investment with Gaussian drift process}

In this section we solve a generic optimal investment problem for a stock with a Gaussian risk premium. We shall see that all the utility maximisation problems of the paper are of this form.

On a probability space $(\Omega, \mathcal{F}, P)$ equipped with a filtration $\mathbb{F}=\left(\mathcal{F}_{t}\right)_{0 \leq t \leq T}$, suppose a stock price $S=\left(S_{t}\right)_{0 \leq t \leq T}$ follows

$$
d S_{t}=\sigma S_{t}\left(\delta_{t} d t+d B_{t}\right)
$$

with $\sigma>0$ constant, $B$ an $\mathbb{F}$-Brownian motion and $\delta=\left(\delta_{t}\right)_{0 \leq t \leq T}$ an $\mathbb{F}$-adapted process. Assume that $\delta$ is given by

$$
\delta_{t}=\delta_{0}+\int_{0}^{t} \mathrm{w}_{s} d B_{s}, \quad 0 \leq t \leq T,
$$

for some $\mathcal{F}_{0}$-measurable Gaussian random variable $\delta_{0}$ independent of $B$, and where w is a deterministic function of time given by

$$
\mathrm{w}_{t}=\frac{\mathrm{w}_{0}}{1+\mathrm{w}_{0} t}, \quad 0 \leq t \leq T,
$$

for some constant $\mathrm{w}_{0}$. Note that $\mathrm{w}$ satisfies the ordinary differential equation (ODE)

$$
\frac{d \mathrm{w}_{t}}{d t}=-\mathrm{w}_{t}^{2}
$$

Note that $\delta$ satisfies the integrability condition

$$
E\left[\int_{0}^{T} \delta_{t}^{2} d t \mid \mathcal{F}_{0}\right]<\infty, \quad \text { a.s. }
$$

Consider optimal investment in $S$ to maximise expected utility of terminal wealth, over $\mathbb{F}$-adapted self-financing portfolios. Let $\theta=\left(\theta_{t}\right)_{0 \leq t \leq T}$ denote an $\mathbb{F}$-adapted trading strategy representing the proportion of wealth invested in the stock. Assume the interest rate is zero, for simplicity. Then the wealth process is $X=\left(X_{t}\right)_{0 \leq t \leq T}$, following

$$
d X_{t}=\sigma \theta_{t} X_{t}\left(\delta_{t} d t+d B_{t}\right), \quad X_{0}=x>0
$$

An $\mathbb{F}$-adapted portfolio process $\theta$ is admissible if we have

$$
\int_{0}^{T} \theta_{t}^{2} d t<\infty, \quad \text { and } X_{t} \geq 0 \text { almost surely, for all } t \in[0, T],
$$

and denote the set of admissible strategies over $[0, T]$ by $\mathcal{A}(T ; \mathbb{F})$.

The utility function $U_{p}: \mathbb{R}^{+} \rightarrow \mathbb{R}$ is of the HARA class, defined by

$$
U_{p}(x):= \begin{cases}x^{p} / p, & p<1, \quad p \neq 0, \\ \log x, & p=0 .\end{cases}
$$

The value function of an agent who uses $\mathbb{F}$-adapted strategies will be defined as $u: \mathbb{R}^{+} \rightarrow \mathbb{R}$, given by

$$
u(x ; T, \mathbb{F}):=\sup _{\theta \in \mathcal{A}(T ; \mathbb{F})} E\left[U_{p}\left(X_{T}\right) \mid \mathcal{F}_{0}\right],
$$

and we write $u(x) \equiv u(x ; T, \mathbb{F})$ when we do not need to emphasise the investment horizon and filtration governing the investment strategies. 
Theorem 1. Assume that $1+\mathrm{w}_{0} T>0$. For $p \neq 0$, define $q$ by $p^{-1}+q^{-1}=1$. For $0<p<1$ assume also that $1+q \mathrm{w}_{0} T>0$. Then the value function in (8) is given by

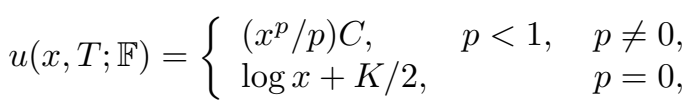

where $C, K$ are $\mathcal{F}_{0}$-measurable random variables given by

$$
\begin{aligned}
C & =\left[\left(1+\mathrm{w}_{0} T\right)^{p}\left(1+q \mathrm{w}_{0} T\right)^{1-p}\right]^{-1 / 2} \exp \left(-\frac{q \delta_{0}^{2} T}{2\left(1+q \mathrm{w}_{0} T\right)}\right), \\
K & =\left(\delta_{0}^{2}+\mathrm{w}_{0}\right) T-\log \left(1+\mathrm{w}_{0} T\right),
\end{aligned}
$$

The optimal $\mathbb{F}$-adapted trading strategy $\theta^{*}$ achieving the supremum in (8) is given by

$$
\theta_{t}^{*}=\left\{\begin{array}{lll}
\delta_{t}\left[\sigma(1-p)\left(1+q \mathrm{w}_{t}(T-t)\right)\right]^{-1}, & p<1, & p \neq 0, \\
\delta_{t} / \sigma, & p=0,
\end{array}\right\} \quad 0 \leq t \leq T .
$$

Proof. For brevity, write $u(x, T ; \mathbb{F}) \equiv u(x)$ in this proof.

With the integrability condition (4) and the stock dynamics (1), we are within the classical framework for portfolio optimisation via convex duality, as surveyed in Karatzas [19], for example.

Let $Q$ denote the unique martingale measure for this market. The change of measure martingale $Z:=\left(Z_{t}\right)_{0 \leq t \leq T}$ is defined by

$$
Z_{t}:=\left.\frac{d Q}{d P}\right|_{\mathcal{F}_{t}}=\mathcal{E}(-\delta \cdot B)_{t}, \quad 0 \leq t \leq T,
$$

and satisfies the SDE

$$
d Z_{t}=-\delta_{t} Z_{t} d B_{t}, \quad Z_{0}=1
$$

Notice that

$$
\lim _{\mathrm{w}_{0} \rightarrow 0} Z_{t}=\mathcal{E}\left(-\delta_{0} B\right)_{t}=\exp \left(-\delta_{0} B_{t}-\frac{1}{2} \delta_{0}^{2} t\right) .
$$

One can verify that $Z$ is given explicitly in terms of $\delta$ by

$$
Z_{t}=\left(\frac{\mathrm{w}_{0}}{\mathrm{w}_{t}}\right)^{1 / 2} \exp \left[-\frac{1}{2}\left(\frac{\delta_{t}^{2}}{\mathrm{w}_{t}}-\frac{\delta_{0}^{2}}{\mathrm{w}_{0}}\right)\right], \quad 0 \leq t \leq T,
$$

since (14) gives a positive $(P, \mathbb{F})$-martingale satisfying (12). ${ }^{1}$ Note also that (14) is indeed welldefined even for $\mathrm{w}_{0} \rightarrow 0$, and that (14) reduces to (13) in the limit $\mathrm{w}_{0} \rightarrow 0$.

Consider the utility maximisation problem (8) when $p<1, p \neq 0$. The proof for $p=0$ follows identical arguments (with more straightforward computations).

Introduce the convex conjugate $\widetilde{U}_{p}$ of the utility function, defined by

$$
\widetilde{U}_{p}(y):=\sup _{x>0}\left[U_{p}(x)-x y\right], \quad y>0 .
$$

For power utility, $p<1, p \neq 0, \widetilde{U}_{p}$ is given by

$$
\widetilde{U}_{p}(y)=-\frac{y^{q}}{q}, \quad \frac{1}{p}+\frac{1}{q}=1 .
$$

\footnotetext{
${ }^{1}$ One way to derive (14) is to make the ansatz $Z_{t}=f\left(t, \delta_{t}\right)$ for a smooth function $f:[0, T] \times \mathbb{R} \rightarrow \mathbb{R}^{+}$. The Itô formula along with the SDE (2) for $\delta$ give

$$
d Z_{t}=\left[f_{t}\left(t, \delta_{t}\right)+\frac{1}{2} \mathrm{w}_{t}^{2} f_{x x}\left(t, \delta_{t}\right)\right] d t+\mathrm{w}_{t} f_{x}\left(t, \delta_{t}\right) d B_{t},
$$

with subscripts of $f$ denoting partial derivatives. Equating (12) and (15) yields partial differential equations for $f:$

$$
\mathrm{w}_{t} f_{x}(t, x)=-x f(t, x), \quad f_{t}(t, x)+\frac{1}{2} \mathrm{w}_{t}^{2} f_{x x}(t, x)=0,
$$

with $f(0, \cdot)=Z_{0}=1$. The solution to these equations gives $Z_{t}$ in the form (14).
} 
The dual value function is defined by

$$
\tilde{u}(y):=E\left[\widetilde{U}_{p}\left(y Z_{T}\right) \mid \mathcal{F}_{0}\right], \quad y>0,
$$

so using (16) is given by

$$
\tilde{u}(y)=-\frac{y^{q}}{q} E\left[Z_{T}^{q} \mid \mathcal{F}_{0}\right] .
$$

By classical results on portfolio optimisation via convex duality ([19], for instance) the primal and dual value functions are conjugate:

$$
\tilde{u}(y)=\sup _{x>0}[u(x)-x y], \quad u(x)=\inf _{y>0}[\tilde{u}(y)+x y] .
$$

Using this and (17), then the primal value function $u$ is indeed given by (9), with $C$ given by

$$
C=\left(E\left[Z_{T}^{q} \mid \mathcal{F}_{0}\right]\right)^{1-p} .
$$

It remains to show that $C$ is equal to the expression in (10), and that the optimal strategy is given by (11).

Once again by classical duality results [19], the optimal terminal wealth $X_{T}^{*}$, attained by adopting the strategy that achieves the supremum in (8), is given by

$$
X_{T}^{*}=-\widetilde{U}_{p}^{\prime}\left(u^{\prime}(x) Z_{T}\right) .
$$

Hence, using the form (9) for $u$, we obtain

$$
X_{T}^{*}=\frac{x}{E\left[Z_{T}^{q} \mid \mathcal{F}_{0}\right]} Z_{T}^{-(1-q)} .
$$

The optimal wealth process $X^{*}$ is a $(Q, \mathbb{F})$-martingale (again, from classical duality results, see Theorem 2.3.2 in [19]), so that

$$
X_{t}^{*}=E^{Q}\left[X_{T}^{*} \mid \mathcal{F}_{t}\right]=\frac{1}{Z_{t}} E\left[Z_{T} X_{T}^{*} \mid \mathcal{F}_{t}\right]=\frac{x}{Z_{t} E\left[Z_{T}^{q} \mid \mathcal{F}_{0}\right]} E\left[Z_{T}^{q} \mid \mathcal{F}_{t}\right], \quad 0 \leq t \leq T,
$$

where $E^{Q}$ denotes expectation under $Q$. So, to compute explicit formulae for $C$ in (18) and the optimal wealth process, we need to evaluate the last conditional expectation in (19).

From (2) and (3), for $t \leq T$, and conditional on $\mathcal{F}_{t}, \delta_{T}$ is Gaussian according to

$$
\operatorname{Law}\left(\delta_{T} \mid \mathcal{F}_{t}\right)=\mathrm{N}\left(\delta_{t}, \mathrm{w}_{t}-\mathrm{w}_{T}\right), \quad 0 \leq t \leq T .
$$

For a Gaussian random variable $Y \sim \mathrm{N}\left(m, s^{2}\right)$, we have, for $c \in \mathbb{R}$,

$$
E \exp \left(c Y^{2}\right)=\frac{1}{\sqrt{1-2 c s^{2}}} \exp \left(\frac{c m^{2}}{1-2 c s^{2}}\right) .
$$

Using this along with the explicit expression (14) for $Z$, we find that $C$ is indeed given by (10). Note that $1+q \mathrm{w}_{0} T>0$ when $0<p<1$ and $1+\mathrm{w}_{0} T>0$ due to the conditions on $\mathrm{w}_{0}$ given in the theorem, so the expression in (10) is well defined.

For the optimal wealth process we obtain the formula

$$
X_{t}^{*}=x\left(\frac{\Psi_{t}}{\Psi_{0}}\right)^{1 / 2} \exp \left(\frac{1}{2}(1-q)\left(\Phi_{t}-\Phi_{0}\right)\right), \quad 0 \leq t \leq T,
$$

where

$$
\Psi_{t}:=\frac{\mathrm{w}_{t}}{1+q \mathrm{w}_{t}(T-t)}, \quad \Phi_{t}:=\frac{\delta_{t}^{2}}{\mathrm{w}_{t}\left(1+q \mathrm{w}_{t}(T-t)\right)}, \quad 0 \leq t \leq T .
$$

To compute the optimal trading strategy $\theta^{*}$, we apply the Itô formula to (20), use the SDE (2) for $\delta$ and the ODE (3) for w, and compare the coefficient of $d B_{t}$ in $d X_{t}^{*}$ with that in (5) for the case of the optimal wealth process. This gives (11). 


\section{The model}

On a complete probability space $(\Omega, \mathcal{F}, P)$ equipped with a filtration $\mathbb{F}:=\left(\mathcal{F}_{t}\right)_{0 \leq t \leq T}$, our model comprises a stock with price process $S=\left(S_{t}\right)_{0 \leq t \leq T}$ following

$$
d S_{t}=\sigma S_{t}\left(\lambda d t+d B_{t}\right)
$$

where $B=\left(B_{t}\right)_{0 \leq t \leq T}$ is a one-dimensional $\mathbb{F}$-Brownian motion. We shall assume the risk premium $\lambda$ is an unknown constant, so we take it to be an $\mathcal{F}_{0}$-measurable Gaussian random variable, independent of $B$. The volatility $\sigma>0$ is assumed to be known. With continuous price monitoring it could be inferred exactly from the quadratic variation of $S$, and we make this approximation to focus on the more severe problem of drift uncertainty. See Rogers [28] for an account of the relative severity of drift versus volatility uncertainty. For simplicity, we take the interest rate to be zero.

Define the process $\xi=\left(\xi_{t}\right)_{0 \leq t \leq T}$ by

$$
\xi_{t}:=\frac{1}{\sigma} \int_{0}^{t} \frac{d S_{s}}{S_{s}}=\lambda t+B_{t}, \quad 0 \leq t \leq T,
$$

which by the Itô formula can be expressed in terms of $S$ :

$$
\xi_{t}=\frac{1}{\sigma} \log \left(\frac{S_{t}}{S_{0}}\right)+\frac{1}{2} \sigma t, \quad 0 \leq t \leq T
$$

and denote by $\widehat{\mathbb{F}}=\left(\widehat{\mathcal{F}}_{t}\right)_{0 \leq t \leq T}$ the filtration generated by $\xi$ :

$$
\widehat{\mathcal{F}}_{t}:=\sigma\left(\xi_{s} ; 0 \leq s \leq t\right), \quad 0 \leq t \leq T .
$$

Then $\widehat{\mathbb{F}}$ coincides with the stock price filtration, and we have $\widehat{\mathcal{F}}_{t} \subseteq \mathcal{F}_{t}$, for all $t \in[0, T]$. We shall sometimes refer to an agent whose information set is $\widehat{\mathbb{F}}$ as a regular agent (or regular trader), to distinguish such an agent from an insider, who will have additional information as well as that provided by the data from $\xi$.

Let $L$ denote an $\mathcal{F}$-measurable random variable. We model an insider as an agent who has knowledge at time zero of the value of $L$, where $L$ will represent (typically, noisy) knowledge of an $\mathcal{F}_{T}$-measurable random variable, either $\xi_{T}$ (equivalently, $S_{T}$ ) or the terminal Brownian motion $B_{T}$. In addition, the insider will not know the value of the risk premium $\lambda$, and her trading strategies will be adapted to the regular observation filtration $\widehat{\mathbb{F}}$ augmented by the inside information, represented by $\sigma(L)$, the sigma-field generated by $L$.

The uncertainty in the random variable $\lambda$ will be modelled by assuming that its prior distribution conditional on $\widehat{\mathcal{F}}_{0}$ is Gaussian, according to the following standing assumption.

Assumption 1. The distribution of $\lambda$ conditional on $\widehat{\mathcal{F}}_{0}$ is Gaussian, independent of $B$, with

$$
E\left[\lambda \mid \widehat{\mathcal{F}}_{0}\right]=E[\lambda]=\lambda_{0}, \quad \operatorname{var}\left[\lambda \mid \widehat{\mathcal{F}}_{0}\right]=\operatorname{var}[\lambda]=\mathrm{v}_{0},
$$

for given constants $\lambda_{0}$ and $\mathrm{v}_{0} \geq 0 .^{2}$

Given the filtration $\mathbb{F}=\left(\mathcal{F}_{t}\right)_{0 \leq t \leq T}$ and the random variable $L$, denote the enlarged filtration by $\mathbb{F}^{L}=\left(\mathcal{F}_{t}^{L}\right)_{0 \leq t \leq T}$, given by

$$
\mathcal{F}_{t}^{L}:=\mathcal{F}_{t} \vee \sigma(L), \quad 0 \leq t \leq T .
$$

Similarly, the insider's observation filtration will be denoted by $\widehat{\mathbb{F}}^{L}:=\left(\widehat{\mathcal{F}}_{t}^{L}\right)_{0 \leq t \leq T}$, given by

$$
\widehat{\mathcal{F}}_{t}^{L}:=\widehat{\mathcal{F}}_{t} \vee \sigma(L), \quad 0 \leq t \leq T,
$$

that is the regular agent's observation filtration enlarged by $\sigma(L)$. We shall sometimes write $\widehat{\mathbb{F}}^{0} \equiv \widehat{\mathbb{F}}$ to signify that the case without inside information may be considered as that with $L \equiv 0$.

\footnotetext{
${ }^{2}$ One way to choose $\lambda_{0}, \mathrm{v}_{0}$ would be to use past data before time zero to obtain a point estimate of $\lambda$, and to use the distribution of the estimator as the prior, as in Monoyios [25].
} 


\subsection{The utility maximisation problems}

The agents in this article will be restricted to using $\widehat{\mathbb{F}}^{L}$-adapted strategies (if there is inside information) or $\widehat{\mathbb{F}}$-adapted strategies if there is no inside information (equivalently, if $L \equiv 0$ ). The utility function $U_{p}$ is given by (7).

With the convention that $L \equiv 0$ corresponds to the situation with no inside information, denote by $\theta^{L}=\left(\theta_{t}^{L}\right)_{0 \leq t \leq T}$ the agent's $\widehat{\mathbb{F}}^{L}$-adapted trading strategy, representing the proportion of wealth invested in the stock. Denote the corresponding wealth process by $X^{L}=\left(X_{t}^{L}\right)_{0 \leq t \leq T}$. The set $\mathcal{A}\left(T ; \widehat{\mathbb{F}}^{L}\right)$ of admissible trading strategies over the horizon $[0, T]$ is defined by the analogue of $(6)$, as

$$
\mathcal{A}\left(T ; \widehat{\mathbb{F}}^{L}\right):=\left\{\theta^{L}: \theta^{L} \text { is } \widehat{\mathbb{F}}^{L} \text {-adapted, } \int_{0}^{T}\left(\theta_{t}^{L}\right)^{2} d t<\infty \text { a.s., with } X_{t}^{L} \geq 0 \text { a.s. } \forall t \in[0, T]\right\} .
$$

Given initial capital $x>0$, the insider's maximal expected utility is given by the value function $u_{L}$, defined by

$$
u_{L}\left(x ; T, \widehat{\mathbb{F}}^{L}\right) \equiv u_{L}(x):=\sup _{\theta^{L} \in \mathcal{A}\left(T ; \widehat{\mathbb{F}}^{L}\right)} E\left[U_{p}\left(X_{T}^{L}\right) \mid \widehat{\mathcal{F}}_{0}^{L}\right]
$$

and we shall write $u_{L}(x) \equiv u_{L}\left(x ; T ; \widehat{\mathbb{F}}^{L}\right)$ when we do not need to emphasise the investment horizon and filtration under consideration. Our goal is to compute the optimal strategy $\theta^{L, *}$ achieving the supremum in (25) as well as the value function $u_{L}$, for three particular cases of $L$, and hence three choices of filtration $\widehat{\mathbb{F}}^{L}$, as listed below.

Problem 0 (Optimal investment with partial information). Here, $L \equiv 0, \widehat{\mathbb{F}}^{L}=\widehat{\mathbb{F}}^{0} \equiv \widehat{\mathbb{F}}$, so there is no inside information. This problem was considered by Rogers [28] among many others. We describe it here (for a wider range of risk aversion parameter than in [28]) to establish some notations and to facilitate subsequent comparisons between the maximal utilities and optimal trading strategies of the insider and the regular trader.

Problem 1 (Investment with Brownian inside information and drift uncertainty). Here, $L$ is given by

$$
L=L_{B}:=a B_{T}+(1-a) \epsilon, \quad 0<a \leq 1,
$$

where $B_{T}$ is the terminal value of the Brownian motion in (22) and $\epsilon$ is a random variable on $(\Omega, \mathcal{F})$ which is standard normal and independent of $B, \lambda$ and $\xi$. The case $a=1$ corresponds to exact information on $B_{T}$, while $a \in(0,1)$ implies noisy information.

The relevant filtration is therefore $\widehat{\mathbb{F}}^{L_{B}}$. For brevity of notation we shall sometimes write $\widehat{\mathbb{F}}^{L_{B}} \equiv \widehat{\mathbb{F}}^{B}$, with a similar convention for other quantities, so the trading strategy will be denoted by $\theta^{B}$, the wealth process by $X^{B}$, the value function by $u_{B}$, and so on.

Problem 2 (Investment with stock price information and drift uncertainty). Here, $L$ is given by

$$
L=L_{S}:=a \xi_{T}+(1-a) \epsilon, \quad 0<a \leq 1,
$$

where $\xi$ is defined in (22) and $\epsilon$ is again standard normal and independent of $B, \lambda$ and $\xi$. The relevant filtration is therefore $\widehat{\mathbb{F}}^{L_{S}}$. For brevity of notation we shall sometimes write $\widehat{\mathbb{F}}^{L_{S}} \equiv \widehat{\mathbb{F}}^{S}$, with a similar convention for other quantities.

\subsection{Outline of methodology}

The approach to solving the optimal investment problems is as follows. We start with the model (21) written in the underlying filtration $\mathbb{F}$. If inside information is available, we form the enlarged filtration $\mathbb{F}^{L}=\left(\mathcal{F}_{t}^{L}\right)_{0 \leq t \leq T}$ given by (23). We write the stock price dynamics with respect to $\mathbb{F}^{L}$, yielding an SDE of the form

$$
d S_{t}=\sigma S_{t}\left(\lambda_{t}^{L} d t+d B_{t}^{L}\right)
$$

where $B^{L}$ is an $\mathbb{F}^{L}$-Brownian motion and $\lambda^{L}$ is an $\mathbb{F}^{L}$-adapted process, with $B^{L}, \lambda^{L}$ given by

$$
B_{t}^{L}=B_{t}-\int_{0}^{t} \nu_{s}^{L} d s, \quad \lambda_{t}^{L}:=\lambda+\nu_{t}^{L}, \quad 0 \leq t \leq T .
$$


Here, $\nu^{L}=\left(\nu_{t}^{L}\right)_{0 \leq t \leq T}$ is an $\mathbb{F}^{L}$-adapted process called the information drift. This is specified using classical enlargement of filtration results $[18,24,30]$ and depends explicitly on the random variable $L$. When there is no inside information, $\nu^{L} \equiv 0$.

If the value of $\lambda$ was known, then (28) would constitute the insider's stock price SDE on the stochastic basis $\left(\Omega, \mathcal{F}_{T}^{L}, \mathbb{F}^{L}, P\right)$. The uncertainty in the value of $\lambda$ means that the agent filters $\lambda^{L}$ (and hence $\lambda$ ) from the stock price observations, suitably augmented by the inside information, that is, given $\widehat{\mathbb{F}}^{L}$. The agent thus infers the conditional expectation

$$
\widehat{\lambda}_{t}^{L}:=E\left[\lambda_{t}^{L} \mid \widehat{\mathcal{F}}_{t}^{L}\right], \quad 0 \leq t \leq T .
$$

The initial (prior) distribution of $\lambda$ given $\widehat{\mathcal{F}}_{0}$ is given by Assumption 1 . We translate this into a prior distribution for $\lambda^{L}$ given $\widehat{\mathcal{F}}_{0}^{L}$, and this also turns out to be Gaussian:

$$
\operatorname{Law}\left(\lambda_{0}^{L} \mid \widehat{\mathcal{F}}_{0}^{L}\right)=\mathrm{N}\left(\widehat{\lambda}_{0}^{L}, V_{0}^{L}\right), \quad \text { independent of } B^{L},
$$

(where $\mathrm{N}(m, V)$ denotes the normal probability law of mean $m$ and variance $V$ ), for some $\widehat{\mathcal{F}}_{0}^{L}$ measurable quantities $\widehat{\lambda}_{0}^{L}$ and $V_{0}^{L} \geq 0$, given in terms of $\lambda_{0}, \mathrm{v}_{0}$, the parameters of the prior for $\lambda$ given in Assumption 1.

To compute $\widehat{\lambda}^{L}=\left(\widehat{\lambda}_{t}^{L}\right)_{0 \leq t \leq T}$ we use a Kalman-Bucy filter, with the SDEs for the signal process $\lambda^{L}$ and the observation process $\xi$ both written with respect to the enlarged filtration $\mathbb{F}^{L}$. These turn out to be linear SDEs, and the Kalman-Bucy algorithm then incorporates both the stock price observations and the inside information into the best estimate of $\lambda^{L}$. We outline in Proposition 3 how the usual Kalman-Bucy equations do indeed hold (with modified initial conditions reflecting the insider information) and do indeed incorporate the inside information, provided the original filtration $\mathbb{F}$ has first been enlarged by $\sigma(L)$ and the signal-observation system SDEs are written with respect to $\mathbb{F}^{L}$. By carrying out enlargement of filtration before filtering, we incorporate the effect of the inside information on the estimation of the unknown risk premium.

The result of the filtering procedure is to convert the partial information model (28) to a full information model written with respect to the stochastic basis $\left(\Omega, \widehat{\mathcal{F}}_{T}^{L}, \widehat{\mathbb{F}}^{L}, P\right)$, of the form

$$
d S_{t}=\sigma S_{t}\left(\widehat{\lambda}_{t}^{L} d t+d \widehat{B}_{t}^{L}\right)
$$

where $\widehat{B}^{L}$ is an $\widehat{\mathbb{F}}^{L}$-Brownian motion and the filtered risk premium $\widehat{\lambda}^{L}$ turns out to be a Gaussian process, specified explicitly by the filtering algorithm. Finally, once we have the completely observable model (30), we are able to solve the utility maximisation problem (25) via a classical dual approach.

\section{Optimal investment with partial information}

In this section we present the solution to Problem 0, in which the agent has no inside information and uses $\widehat{\mathbb{F}}$-adapted strategies to maximise expected utility of terminal wealth. We include this well-known material here to establish some notation and provide results for comparison between the problems with and without privileged information.

Prior to filtering, the stock price follows $(21)$ on the stochastic basis $(\Omega, \mathcal{F}, \mathbb{F}, P)$. We acknowledge the agent's uncertainty concerning the value of the risk premium $\lambda$ by treating it as an unknown constant, hence a random variable, with prior distribution given in Assumption 1. Adopting a filtering framework and considering $\lambda$ as an unobservable signal process, it follows the trivial SDE

$$
d \lambda=0
$$

and the observation process is $\xi$, given by $(22)$.

Define the conditional mean and variance of $\lambda$ given $\widehat{\mathbb{F}}$ by

$$
\widehat{\lambda}_{t}:=E\left[\lambda \mid \widehat{\mathcal{F}}_{t}\right], \quad \mathrm{v}_{t}:=E\left[\left(\lambda-\widehat{\lambda}_{t}\right)^{2} \mid \widehat{\mathcal{F}}_{t}\right]=E\left[\left(\lambda-\widehat{\lambda}_{t}\right)^{2}\right], \quad 0 \leq t \leq T,
$$

and $\mathrm{v}:[0, T] \rightarrow \mathbb{R}^{+}$will be a deterministic function of time (as usual with a Kalman filter) independent of $\widehat{\mathbb{F}}$. We then have the following solution to Problem 0 . 
Proposition 1 (Solution to Problem 0). For $0<p<1$, assume that $1+q \mathrm{v}_{0} T>0$, where $p^{-1}+q^{-1}=1$. The value function in (25) when there is no inside information (so $L \equiv 0$ ) and $\widehat{\mathbb{F}}^{L}=\widehat{\mathbb{F}}^{0} \equiv \widehat{\mathbb{F}}$ is given by

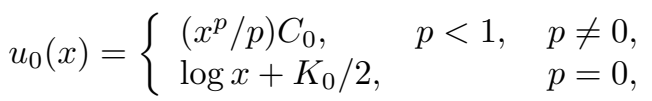

where $C_{0}, K_{0}$ are constants given by

$$
\begin{aligned}
& C_{0}=\left[\left(1+\mathrm{v}_{0} T\right)^{p}\left(1+q \mathrm{v}_{0} T\right)^{1-p}\right]^{-1 / 2} \exp \left[-\frac{q \lambda_{0}^{2} T}{2\left(1+q \mathrm{v}_{0} T\right)}\right], \quad \frac{1}{p}+\frac{1}{q}=1, \\
& K_{0}=\left(\lambda_{0}^{2}+\mathrm{v}_{0}\right) T-\log \left(1+\mathrm{v}_{0} T\right) .
\end{aligned}
$$

The optimal $\widehat{\mathbb{F}}$-adapted trading strategy achieving the supremum in (25) is $\theta^{0, *}$, given by

$$
\theta_{t}^{0, *}=\left\{\begin{array}{ll}
\widehat{\lambda}_{t}\left[\sigma(1-p)\left(1+q \mathrm{v}_{t}(T-t)\right)\right]^{-1}, & p<1, \\
\widehat{\lambda}_{t} / \sigma, & p \neq 0, \\
& p=0,
\end{array}\right\} \quad 0 \leq t \leq T
$$

where $\widehat{\lambda}, \mathrm{v}$ are given by

$$
\widehat{\lambda}_{t}=\frac{\lambda_{0}+\mathrm{v}_{0} \xi_{t}}{1+\mathrm{v}_{0} t}, \quad \mathrm{v}_{t}=\frac{\mathrm{v}_{0}}{1+\mathrm{v}_{0} t}, \quad 0 \leq t \leq T,
$$

and $\xi$ is defined in (22).

Proof. By the Kalman-Bucy filter (Theorem 10.3 in Lipster and Shiryaev [23] or Theorem V.9.2 in Fleming and Rishel [14]), $\widehat{\lambda}$ satisfies the SDE

$$
d \widehat{\lambda}_{t}=\mathrm{v}_{t}\left(d \xi_{t}-\widehat{\lambda}_{t} d t\right)=\mathrm{v}_{t} d \widehat{B}_{t}, \quad \widehat{\lambda}_{0}=\lambda_{0},
$$

where $\widehat{B}$ is the innovations process, an $\widehat{\mathbb{F}}$-Brownian motion defined in terms of $\xi, \widehat{\lambda}$ by

$$
\widehat{B}_{t}:=\xi_{t}-\int_{0}^{t} \widehat{\lambda}_{s} d s, \quad 0 \leq t \leq T,
$$

and the conditional variance $\mathrm{v}$ satisfies the deterministic Riccati equation

$$
\frac{d \mathrm{v}_{t}}{d t}=-\mathrm{v}_{t}^{2},
$$

with initial value $\mathrm{v}_{0}$ and solution as given in (34). The solution to (35) is the Gaussian process

$$
\widehat{\lambda}_{t}=\lambda_{0}+\int_{0}^{t} \mathrm{v}_{s} d \widehat{B}_{s}, \quad 0 \leq t \leq T,
$$

and in terms of $\xi$, the solution to (35) for $\widehat{\lambda}$ is as given in (34).

The stock price SDE is $d S_{t}=\sigma S_{t} d \xi_{t}$. With respect to $\widehat{\mathbb{F}}$, we use (36) to write this as

$$
d S_{t}=\sigma S_{t}\left(\widehat{\lambda}_{t} d t+d \widehat{B}_{t}\right) .
$$

From (38) it is easy to see that we have $E\left[\int_{0}^{T} \widehat{\lambda}_{t}^{2} d t \mid \widehat{\mathcal{F}}_{0}\right]<\infty$, a.s. This places us, with the dynamics in (39), firmly within a classical full information framework for portfolio optimisation via convex duality, and the results in the theorem then follow from Theorem 1 with $(\widehat{\mathbb{F}}, \widehat{B}, \widehat{\lambda}, \mathrm{v})$ in place of $(\mathbb{F}, B, \delta, \mathrm{w})$.

Naturally, in the limit $\mathrm{v}_{0} \rightarrow 0$, the risk premium of the stock becomes the constant $\lambda_{0}$ and Proposition 1 gives the solution to the classical full information Merton optimal investment problem for a stock with constant risk premium $\lambda_{0}$ and volatility $\sigma$. 


\section{Optimal investment with inside information and drift uncertainty}

In this section we give the main results, the solutions to Problems 1 and 2 in which the agent is an insider with knowledge at time zero of an $\mathcal{F}$-measurable random variable $L$, and who does not know the value of the risk premium $\lambda$. We then make some comparisons between the maximal utilities and optimal trading strategies of the insider and the regular trader. The proofs are given in Section 7, after establishing some enlargement of filtration and filtering results in the next section.

Define the modulated terminal time $T_{a}$ by

$$
T_{a}:=T+\left(\frac{1-a}{a}\right)^{2}, \quad 0<a \leq 1
$$

which will appear frequently, and note that $\lim _{a \rightarrow 1} T_{a}=T$.

\subsection{Brownian inside information}

In Problem 1 the insider has access to information on the terminal Brownian motion $B_{T}$. The random variable $L$ is $L_{B}$ given in (26). For brevity of notation we shall often write $\widehat{\mathbb{F}}^{L_{B}} \equiv \widehat{\mathbb{F}}^{B}$, with a similar convention for the value function, $u_{L_{B}} \equiv u_{B}$, and other relevant quantities.

Theorem 2 (Solution to Problem 1). For $0<p<1$, assume that

$$
\mathrm{v}_{0} T<\frac{1-p}{p}+\frac{T}{T_{a}} .
$$

The value function of the insider in (25) when the additional information is noisy knowledge of $B_{T}$, so $\widehat{\mathbb{F}}^{L}=\widehat{\mathbb{F}}^{L}{ }_{B} \equiv \widehat{\mathbb{F}}^{B}$, with

$$
L=L_{B}:=a B_{T}+(1-a) \epsilon, \quad 0<a<1,
$$

is given by

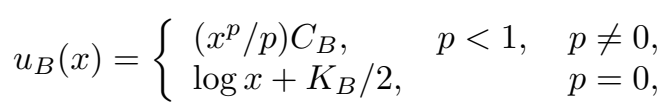

where $C_{B}, K_{B}$ are $\widehat{\mathcal{F}}_{0}^{B}$-measurable random variables given by

$$
\begin{aligned}
C_{B} & =\left[\left(1+\mathrm{v}_{0}^{B} T\right)^{p}\left(1+q \mathrm{v}_{0}^{B} T\right)^{1-p}\right]^{-1 / 2} \exp \left(-\frac{q\left(\widehat{\lambda}_{0}^{B}\right)^{2} T}{2\left(1+q \mathrm{v}_{0}^{B} T\right)}\right), \frac{1}{p}+\frac{1}{q}=1, \\
K_{B} & =\left(\left(\widehat{\lambda}_{0}^{B}\right)^{2}+\mathrm{v}_{0}^{B}\right) T-\log \left(1+\mathrm{v}_{0}^{B} T\right),
\end{aligned}
$$

and where $\widehat{\lambda}_{0}^{B}, \mathrm{v}_{0}^{B}$ are given by

$$
\widehat{\lambda}_{0}^{B}=\lambda_{0}+\frac{L_{B}}{a T_{a}}, \quad \mathrm{v}_{0}^{B}=\mathrm{v}_{0}-\frac{1}{T_{a}} .
$$

The optimal $\widehat{\mathbb{F}}^{B}$-adapted trading strategy achieving the supremum in (25) is $\theta^{B, *}$, given by

$$
\theta_{t}^{B, *}=\left\{\begin{array}{ll}
\widehat{\lambda}_{t}^{B}\left[\sigma(1-p)\left(1+q \mathrm{v}_{t}^{B}(T-t)\right)\right]^{-1}, & p<1, \\
\widehat{\lambda}_{t}^{B} / \sigma, & p \neq 0, \\
& p=0,
\end{array}\right\} \quad 0 \leq t \leq T,
$$

where $\widehat{\lambda}^{B}, \mathrm{v}^{B}$ are given by

$$
\widehat{\lambda}_{t}^{B}=\frac{\widehat{\lambda}_{0}^{B}+\mathrm{v}_{0}^{B} \xi_{t}}{1+\mathrm{v}_{0}^{B} t}, \quad \mathrm{v}_{t}^{B}=\frac{\mathrm{v}_{0}^{B}}{1+\mathrm{v}_{0}^{B} t}, \quad 0 \leq t \leq T .
$$

For $a=1$ and $\mathrm{v}_{0}>0$ the above results still hold, while for $a=1$ and $\mathrm{v}_{0}=0$ the value function is unbounded for $p \in[0,1)$ and equal to zero for $p<0$. 
The proof will be given in Section 7 .

Naturally, the value function and optimal strategy depend on the random variable $L \equiv L_{B}$. There are clear similarities in the structure of the solution to this problem with that of Problem 0 , with $(\widehat{\lambda}, \mathrm{v})$ replaced by $\left(\widehat{\lambda}_{0}^{B}, \mathrm{v}^{B}\right)$. It turns out that $\mathrm{v}^{B}$ is related to (but not identical to) the conditional variance of the insider's unknown risk premium $\lambda^{B}$ given $\widehat{\mathbb{F}}^{B}$, as we shall see later.

Note that, formally, the correct results for the limiting cases $a=1, \mathrm{v}_{0}>0$ and $a=1, \mathrm{v}_{0}=0$ can be obtained by taking these limits in the value function formula for $a \in(0,1)$, though this does not constitute a proof, and we shall need some more involved arguments to rigorously establish the results for these limiting cases.

For $a=1$ and $\mathrm{v}_{0}>0$ the value function and optimal strategy are well defined, even though the insider has exact knowledge of the value of terminal Brownian motion $B_{T}$. This is to be contrasted with the results of Pikovsky and Karatzas [27], in which there is no drift parameter uncertainty (corresponding to $\mathrm{v}_{0} \rightarrow 0$ here). In [27], exact knowledge of any kind at time zero leads to unbounded logarithmic utility. Here, in contrast, provided $\mathrm{v}_{0}>0$, the parameter uncertainty means that exact Brownian inside information does not lead to an explosion in utility (for $0 \leq p<1$ ) or to zero value function (the maximum possible expected utility) for $p<0$.

But for $\mathrm{v}_{0} \rightarrow 0$ we should, and do, recover results consistent with [27], with unbounded utility for $p \in[0,1)$ and zero for $p<0$. In this case, since $\xi_{T}=B_{T}+\lambda T$, then when the value of $\lambda$ is known with certainty, exact Brownian inside information is equivalent to exact terminal stock price information, and hence Pikovsky and Karatzas [27] find that exact information of any kind leads to unbounded logarithmic utility.

In contrast, we shall see shortly that, with $\mathrm{v}_{0}>0$, only exact terminal stock price knowledge leads to unbounded logarithmic utility. The intuition here is that, with the initial and terminal stock prices known at time zero, one immediately obtains the best possible estimate of $\lambda$ at time zero, and the parameter uncertainty is eliminated as much as is possible from the outset, leading to a utility explosion, and to the filtering algorithm being rendered redundant, as we shall see in the proof of Problem 2.

\subsubsection{Additional utility of the insider}

To quantify the additional utility of the insider with Brownian information relative to the regular trader, let us define the value of the additional information as $\pi_{B}$, given implicitly by

$$
E\left[u_{B}(x) \mid \widehat{\mathcal{F}}_{0}\right]=u_{0}\left(x+\pi_{B}(x)\right) .
$$

In other words, $\pi_{B}$ is the additional wealth needed by the regular trader to achieve, on average, the same expected utility as the insider who knows the value of $L_{B}$. To compute $\pi_{B}$, we need the distribution of $\widehat{\lambda}_{0}^{B}$ given $\widehat{\mathcal{F}}_{0}$. But $\widehat{\mathcal{F}}_{0}$ is in fact the trivial sigma-field, so we have $\operatorname{Law}\left(\widehat{\lambda}_{0}^{B} \mid \widehat{\mathcal{F}}_{0}\right)=$ $\operatorname{Law}\left(\widehat{\lambda}_{0}^{B}\right)$, given by

$$
\widehat{\lambda}_{0}^{B} \sim \mathrm{N}\left(\lambda_{0}, 1 / T_{a}\right)=\mathrm{N}\left(\lambda_{0}, \mathrm{v}_{0}-\mathrm{v}_{0}^{B}\right) .
$$

When $0<p<1$, we assume that $1+q \mathrm{v}_{0} T>0$, so that $u_{0}$ is well-defined. Under this condition we also have $1+q \mathrm{v}_{0}^{B} T>0$, so that $u_{B}$ is also well-defined. Hence $\pi_{B}$ is well-defined and given by

$$
\pi_{B}(x) / x=\left[\left(1+\mathrm{v}_{0} T\right)\left(1+q \mathrm{v}_{0}^{B} T\right)\right]^{1 / 2}\left[\left(1+\mathrm{v}_{0}^{B} T\right)\left(1+q \mathrm{v}_{0} T\right)\right]^{-1 / 2}-1, \quad p<1 .
$$

It can be verified that $\pi_{B}(x)>0$, reflecting the insider's additional expected utility, and that (formally) $\pi_{B}(x) \rightarrow \infty$ (uniformly in $x$ ) for $a=1, \mathrm{v}_{0}=0$, for all values of $p<1$.

\subsection{Stock price inside information}

When the insider has terminal stock price inside information at time zero, given by the random variable $L \equiv L_{S}$ in (27), the solution to the optimal investment problem is as follows.

Theorem 3 (Solution to Problem 2). For $0<p<1$, assume that

$$
\left[1-\left(1+\frac{1}{p}\right) \frac{T}{T_{a}}\right] \mathrm{v}_{0} T<\frac{1-p}{p}+\frac{T}{T_{a}} .
$$


The value function of the insider in (25) when the additional information is noisy knowledge of $\xi_{T}$, so $\widehat{\mathbb{F}}^{L}=\widehat{\mathbb{F}}^{L_{S}} \equiv \widehat{\mathbb{F}}^{S}$, with

$$
L=L_{S}:=a \xi_{T}+(1-a) \epsilon, \quad 0<a<1,
$$

is given by

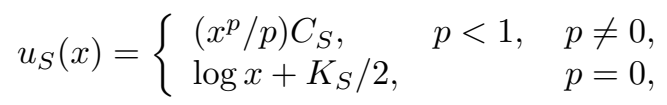

where $C_{S}, K_{S}$ are $\widehat{\mathcal{F}}_{0}^{S}$-measurable random variables given by

$$
\begin{aligned}
C_{S} & =\left[\left(1+\mathrm{v}_{0}^{S} T\right)^{p}\left(1+q \mathrm{v}_{0}^{S} T\right)^{1-p}\right]^{-1 / 2} \exp \left(-\frac{q\left(\widehat{\lambda}_{0}^{S}\right)^{2} T}{2\left(1+q \mathrm{v}_{0}^{S} T\right)}\right), \quad \frac{1}{p}+\frac{1}{q}=1, \\
K_{S} & =\left(\left(\widehat{\lambda}_{0}^{S}\right)^{2}+\mathrm{v}_{0}^{S}\right) T-\log \left(1+\mathrm{v}_{0}^{S} T\right),
\end{aligned}
$$

and where $\widehat{\lambda}_{0}^{S}, \mathrm{v}_{0}^{S}$ are given by

$$
\widehat{\lambda}_{0}^{S}=\frac{\lambda_{0}\left(1-T / T_{a}\right)+\left(1+\mathrm{v}_{0} T\right)\left(L_{S} /\left(a T_{a}\right)\right)}{1+\mathrm{v}_{0} T\left(T / T_{a}\right)}, \quad \mathrm{v}_{0}^{S}=\frac{\left(1-T / T_{a}\right)^{2} \mathrm{v}_{0}}{1+\mathrm{v}_{0} T\left(T / T_{a}\right)}-\frac{1}{T_{a}} .
$$

The optimal $\widehat{\mathbb{F}}^{S}$-adapted trading strategy achieving the supremum in (25) is $\theta^{S, *}$, given by

$$
\theta_{t}^{S, *}=\left\{\begin{array}{ll}
\widehat{\lambda}_{t}^{S}\left[\sigma(1-p)\left(1+q \mathrm{v}_{t}^{S}(T-t)\right)\right]^{-1}, & p<1, \\
\widehat{\lambda}_{t}^{S} / \sigma, & p \neq 0, \\
& p=0,
\end{array}\right\} \quad 0 \leq t \leq T
$$

where $\widehat{\lambda}^{S}, \mathrm{v}^{S}$ are given by

$$
\widehat{\lambda}_{t}^{S}=\frac{\widehat{\lambda}_{0}^{S}+\mathrm{v}_{0}^{S} \xi_{t}}{1+\mathrm{v}_{0}^{S} t}, \quad \mathrm{v}_{t}^{S}=\frac{\mathrm{v}_{0}^{S}}{1+\mathrm{v}_{0}^{S t} t}, \quad 0 \leq t \leq T .
$$

For $a=1$ the value function is unbounded for $p \in[0,1)$ and equal to zero for $p<0$.

The proof will be given in Section 7 .

Again, we observe the similarity in the structure of the solution to this problem with that of Problems 0 and 1 , with $\left(\widehat{\lambda}^{S}, \mathrm{v}^{S}\right)$ in place of $(\widehat{\lambda}, \mathrm{v})$ or $\left(\widehat{\lambda}^{B}, \mathrm{v}^{B}\right)$.

Formally letting $a \rightarrow 1$ in the formula for the value function again leads to the correct conclusion, namely infinite additional utility, though this is not a proof, and we will need careful arguments to establish the result.

\subsubsection{Additional utility of the insider}

We can quantify the additional utility of the insider with terminal stock price information, as we did for the insider with Brownian information. The distribution of $\widehat{\lambda}_{0}^{S}$ in this case is given by

$$
\widehat{\lambda}_{0}^{S} \sim \mathrm{N}\left(\lambda_{0}, \frac{\left(1+\mathrm{v}_{0} T\right)^{2}}{T_{a}\left(1+\mathrm{v}_{0} T\left(T / T_{a}\right)\right)}\right)=\mathrm{N}\left(\lambda_{0}, \mathrm{v}_{0}-\mathrm{v}_{0}^{S}\right) .
$$

When $0<p<1$, we assume that $1+q \mathrm{v}_{0} T>0$, so that $u_{0}$ is well-defined. Under this condition we also have $1+q \mathrm{v}_{0}^{S} T>0$, so that $u_{S}$ is also well-defined. Hence the value of the additional information, $\pi_{S}$, is well-defined and given by

$$
\pi_{S}(x) / x=\left[\left(1+\mathrm{v}_{0} T\right)\left(1+q \mathrm{v}_{0}^{S} T\right)\right]^{1 / 2}\left[\left(1+\mathrm{v}_{0}^{S} T\right)\left(1+q \mathrm{v}_{0} T\right)\right]^{-1 / 2}-1, \quad p<1 .
$$

Once again, it can be verified that $\pi_{S}(x)>0$, reflecting the insider's additional expected utility, and that (formally) $\pi_{S}(x) \rightarrow \infty$ (uniformly in $x$ ) for $a \rightarrow 1$, for all values of $p<1$.

More pertinently, if we compare the values of stock price and Brownian information, we find that for $a \neq 1$,

$$
\pi_{S}(x)>\pi_{B}(x), \text { for all } x,
$$

since we have $\mathrm{v}_{0}^{S}<\mathrm{v}_{0}^{B}$. In other words, stock price information is more valuable than Brownian information when the drift of the stock is unknown. This makes sense, as we need stock price observations to estimate the drift. 


\subsection{Comparison of trading strategies of regular trader and insider}

One can also carry out a comparison of how aggressive the insider is in taking a stock position relative to the regular trader. One way to make a meaningful comparison of $\widehat{\mathbb{F}}^{L}$-adapted strategies with $\widehat{\mathbb{F}}$-adapted strategies, is to condition the insider's optimal portfolio $\theta_{t}^{L}$ on $\widehat{\mathcal{F}}_{t}$.

For $0<p<1$ we always assume that $1+q \mathrm{v}_{0} T>$, and this guarantees that $1+q \mathrm{v}_{0}^{L} T>0$, so that $R^{L}$, defined by

$$
R_{t}^{L}:=\frac{E\left[\theta_{t}^{L, *} \mid \widehat{\mathcal{F}}_{t}\right]}{\theta_{t}^{0, *}}, \quad L \in\{B, S\}, \quad 0 \leq t \leq T,
$$

exists.

The effect of conditioning $\theta^{L, *}$ with respect to $\widehat{\mathbb{F}}$ isolates the multiplier $\left(1+q \mathrm{v}_{t}^{L}(T-t)\right)^{-1}$. This measures the extent to which the insider's stock position is magnified or reduced in response to her estimate $\widehat{\lambda}^{L}$ of the risk premium.

Proposition 2. For $p=0, R_{B}=R_{S}=1, \widehat{\mathbb{F}}$-a.s., while for $p \neq 0$, we have

$$
R_{t}^{B}=\frac{1+q \mathrm{v}_{t}(T-t)}{1+q \mathrm{v}_{t}^{B}(T-t)}, \quad R_{t}^{S}=\frac{1+q \mathrm{v}_{t}(T-t)}{1+q \mathrm{v}_{t}^{S}(T-t)}, \quad 0 \leq t \leq T .
$$

Note that $\mathrm{v}_{t}^{S}<\mathrm{v}_{t}^{B}<\mathrm{v}_{t}$ for $t \in[0, T]$. So for $p \neq 0$ we have the ordering (recall $q<0$ when $p \in(0,1))$

$$
q R_{t}^{S}>q R_{t}^{B}>q, \quad 0 \leq t \leq T .
$$

The insider takes a more aggressive holding in the stock than the regular trader when $p<0$ (corresponding to relative risk aversion larger than 1). This effect is more marked when the inside information is on the stock price rather than the Brownian motion, because in this case the insider derives greater confidence in his estimate of the stock drift than with Brownian knowledge. This result seems intuitively plausible.

When $p=0$ this effect is nullified. Intriguingly, when $p \in(0,1)$, this effect is reversed. In other words, when risk aversion becomes sufficiently small (and, as we have seen, rather complicated conditions on the prior variance are needed for a well-posed problem) an agent with progressively less uncertainty in her estimate of the stock's risk premium, nevertheless takes a less aggressive holding in the stock. This unexpected result, which stems from a partial information framework, has (to the best of our knowledge) not been previously observed.

We need to prove some lemmas on the way to proving Proposition 2.

Lemma 1. Conditional on $\widehat{\mathcal{F}}_{t}, 0 \leq t \leq T$, the distribution of $\xi_{T}$ is given by

$$
\operatorname{Law}\left(\xi_{T} \mid \widehat{\mathcal{F}}_{t}\right)=\mathrm{N}\left(\xi_{t}+\widehat{\lambda}_{t}(T-t),\left(1+\mathrm{v}_{t}(T-t)\right)(T-t)\right), \quad 0 \leq t \leq T .
$$

Proof. Using (36) we have, for $t \leq T$,

$$
\xi_{T}=\xi_{t}+\int_{t}^{T} \widehat{\lambda}_{s} d s+\widehat{B}_{T}-\widehat{B}_{t}
$$

Note that $\xi$ is Gaussian. Using the solution (38) for $\widehat{\lambda}$ we obtain

$$
\int_{t}^{T} \widehat{\lambda}_{s} d s=\widehat{\lambda}_{t}(T-t)+\int_{t}^{T} \mathrm{v}_{s}(T-s) d \widehat{B}_{s}
$$

so that

$$
\xi_{T}=\xi_{t}+\widehat{\lambda}_{t}(T-t)+\int_{t}^{T}\left(1+\mathrm{v}_{s}(T-s)\right) d \widehat{B}_{s}
$$

The result for the mean is immediate, and the variance follows from the Itô isometry and the formula in (34) for $\mathrm{v}$. 
Corollary 1. The information drift $\nu^{L} \equiv \nu^{B} \equiv \nu^{S}$ for both Brownian and stock price inside information satisfies

$$
E\left[\nu_{t}^{L} \mid \widehat{\mathcal{F}}_{t}\right]=0, \quad 0 \leq t \leq T .
$$

Proof. In both Examples 1 and 2, the information drift may be written as

$$
\nu_{t}^{L}=\frac{1}{a\left(T_{a}-t\right)}\left(a\left(\xi_{T}-\xi_{t}-\lambda(T-t)\right)+(1-a) \epsilon\right) .
$$

Recall that $\epsilon \sim \mathrm{N}(0,1)$, independent of $\widehat{\mathbb{F}}$. So, conditioning on $\widehat{\mathcal{F}}_{t}$, recalling that $\widehat{\lambda}_{t}:=E\left[\lambda \mid \widehat{\mathcal{F}}_{t}\right]$ and using Lemma 1, the result follows.

Proof of Proposition 2. Using the definition of $\widehat{\lambda}_{t}^{B}$, the tower property, and Corollary 1, we obtain

$$
E\left[\widehat{\lambda}_{t}^{B} \mid \widehat{\mathcal{F}}_{t}\right]=E\left[E\left[\lambda_{t}^{B} \mid \widehat{\mathcal{F}}_{t}^{B}\right] \mid \widehat{\mathcal{F}}_{t}\right]=E\left[\lambda_{t}^{B} \mid \widehat{\mathcal{F}}_{t}\right]=E\left[\lambda+\nu_{t}^{B} \mid \widehat{\mathcal{F}}_{t}\right]=\widehat{\lambda}_{t}
$$

Similarly, using the tower property and Lemma 1, we find that

$$
E\left[\widehat{\lambda}_{t}^{S} \mid \widehat{\mathcal{F}}_{t}\right]=\widehat{\lambda}_{t},
$$

where we have again used Corollary 1 . The proposition follows easily from (46) and (47).

\section{Enlargement of filtration and filtering}

\subsection{Initial enlargements of filtrations}

In this section we apply classical enlargement of filtration formula to augment a filtration $\mathbb{F}$ (containing a Brownian filtration) with the information carried by an $\mathcal{F}$-measurable random variable $L$. The enlargement formula gives the semi-martingale decomposition of the $\mathbb{F}$-Brownian motion $B$ with respect to the enlarged filtration $\mathbb{F}^{L}$, defined in (23). We give two applications of the enlargement formula, for $L=L_{B}$ in (26) and $L=L_{S}$ in (27), that pertain to our two optimal investment problems with privileged information.

We begin with the filtration $\mathbb{F}=\left(\mathcal{F}_{t}\right)_{0 \leq t \leq T}$ that contains the filtration of the Brownian motion $B$ driving the stock price process in $(21)$, and also $\sigma(\lambda) \in \mathcal{F}_{0}$. Let $L$ be an $\mathcal{F}$-measurable random variable.

Given any bounded Borel function $f: \mathbb{R} \rightarrow \mathbb{R}$, define $\mu(f)=\left(\mu_{t}(f)\right)_{0 \leq t \leq T}$ as the continuous version of the martingale $\left(E\left[f(L) \mid \mathcal{F}_{t}\right]\right)_{0 \leq t \leq T}$. By the representation property of Brownian martingales there exists an $\mathbb{F}$-previsible process $\dot{\mu}(f)=\left(\dot{\mu}_{t}(f)\right)_{0 \leq t \leq T}$ such that

$$
\mu_{t}(f)=E[f(L)]+\int_{0}^{t} \dot{\mu}_{s}(f) d B_{s}, \quad 0 \leq t \leq T .
$$

The enlargement decomposition formula is given by Theorem 12.1 in Yor [30] in the following fashion.

Theorem 4 ([30], Theorem 12.1). Suppose there exists a predictable family of measures $\left(\mu_{t}(d x)\right)_{0 \leq t \leq T}$ such that

$$
\mu_{t}(f)=\int_{\mathbb{R}} f(x) \mu_{t}(d x), \quad 0 \leq t \leq T .
$$

Assume there exists a predictable family $\left(\dot{\mu}_{t}(d x)\right)_{0 \leq t \leq T}$ of measures such that

$$
\text { dt a.s. } \quad \dot{\mu}_{t}(f)=\int_{\mathbb{R}} f(x) \dot{\mu}_{t}(d x), \quad 0 \leq t \leq T,
$$

and such that $d t d P$ a.s. $\dot{\mu}_{t}(d x)$ is absolutely continuous with respect to $\mu_{t}(d x)$. Define $\rho(x, t)$ by

$$
\dot{\mu}_{t}(d x)=\mu_{t}(d x) \rho(x, t) .
$$


Then, provided that

$$
\int_{0}^{t}|\rho(x, s)| d s<\infty, \quad \text { a.s. }
$$

the $\mathbb{F}$-Brownian motion $B$ decomposes as

$$
B_{t}=B_{t}^{L}+\int_{0}^{t} \rho(L, t) d s, \quad 0 \leq t \leq T
$$

with $B^{L}$ and $\mathbb{F}^{L}$-Brownian motion, and the process

$$
\nu_{t}^{L}:=\rho(L, t), \quad 0 \leq t \leq T,
$$

is called the information drift.

Example 1. Take $L=L_{B}$ given in (26). Then $\operatorname{Law}\left[L_{B} \mid \mathcal{F}_{t}\right]=\mathrm{N}\left(a B_{t}, a^{2}\left(T_{a}-t\right)\right)$, where $T_{a}$ is defined in (40). Therefore

$$
\mu_{t}(f)=E\left[f(L) \mid \mathcal{F}_{t}\right]=\frac{1}{a \sqrt{2 \pi\left(T_{a}-t\right)}} \int_{\mathbb{R}} f(x) \exp \left(\frac{\left(x-a B_{t}\right)^{2}}{2 a^{2}\left(T_{a}-t\right)}\right) d x=: \int_{\mathbb{R}} f(x) \mu_{t}(d x) .
$$

Using the Itô formula inside the integral and reversing the order of integration yields

$$
\dot{\mu}_{t}(f)=\frac{1}{a \sqrt{2 \pi\left(T_{a}-t\right)}} \int_{\mathbb{R}} f(x)\left(\frac{x-a B_{t}}{a\left(T_{a}-t\right)}\right) \exp \left(\frac{\left(x-a B_{t}\right)^{2}}{2 a^{2}\left(T_{a}-t\right)}\right) d x=: \int_{\mathbb{R}} f(x) \dot{\mu}_{t}(d x),
$$

and note the measures $\mu_{t}(d x)$ and $\dot{\mu}_{t}(d x)$ do indeed satisfy the conditions given in Theorem 4 . In this case, $\rho(x, t)$ is given by

$$
\rho(x, t)=\frac{x-a B_{t}}{a\left(T_{a}-t\right)}, \quad 0 \leq t \leq T,
$$

and satisfies (48). Therefore, the semimartingale decomposition of $B$ with respect to $\mathbb{F}^{L}=\mathbb{F}^{L_{B}} \equiv$ $\mathbb{F}^{B}$ is

$$
B_{t}=B_{t}^{B}+\int_{0}^{t} \frac{L_{B}-a B_{s}}{a\left(T_{a}-s\right)} d s
$$

with $L_{B}$ as in (26), and where we have written $\mathbb{F}^{L_{B}} \equiv \mathbb{F}^{B}$ and $B^{L_{B}} \equiv B^{B}$ to ease notation.

The information drift in this case is therefore $\nu^{L_{B}} \equiv \nu^{B}$ given by

$$
\nu_{t}^{B}=\frac{L_{B}-a B_{t}}{a\left(T_{a}-t\right)}=\frac{a\left(B_{T}-B_{t}\right)+(1-a) \epsilon}{a\left(T_{a}-t\right)}, \quad 0 \leq t \leq T,
$$

where we have used the explicit form (26) for $L_{B}$.

For $a=1$ we have $L_{B}=B_{T}$ and we obtain the well known Brownian bridge decomposition of $B$ under $\mathbb{F}^{B_{T}}$ :

$$
B_{t}=B_{t}^{B_{T}}+\int_{0}^{t} \frac{B_{T}-B_{s}}{T-s} d s, \quad 0 \leq t \leq T .
$$

Example 2. Take $L=L_{S}$ given in (27). Using $\xi_{T}=B_{T}+\lambda T$, we may write

$$
L_{S}=a\left(B_{T}+\lambda T\right)+(1-a) \epsilon, \quad 0<a \leq 1,
$$

and since $\lambda$ is assumed to be $\mathcal{F}_{0}$-measurable, we have $\operatorname{Law}\left[L_{S} \mid \mathcal{F}_{t}\right]=\mathrm{N}\left(a\left(B_{t}+\lambda T\right), a^{2}\left(T_{a}-t\right)\right)$. Then the same method as in Example 1 yields that the semimartingale decomposition of $B$ with respect to $\mathbb{F}^{L}=\mathbb{F}^{L_{S}} \equiv \mathbb{F}^{S}$ is

$$
B_{t}=B_{t}^{S}+\int_{0}^{t} \frac{L_{S}-a\left(B_{s}+\lambda T\right)}{a\left(T_{a}-s\right)} d s,
$$

with $L_{S}$ as in (27), and where we have written $B^{L_{S}} \equiv B^{S}$ to ease notation. 
Using (52) in the decomposition (53) we may write the information drift in this case as $\nu^{L_{S}} \equiv \nu^{S}$ given by

$$
\nu_{t}^{S}=\frac{L_{S}-a\left(B_{t}+\lambda T\right)}{a\left(T_{a}-t\right)}=\frac{a\left(B_{T}-B_{t}\right)+(1-a) \epsilon}{a\left(T_{a}-t\right)}, \quad 0 \leq t \leq T,
$$

which we see is identical to the information drift in (50) for the case with Brownian inside information. The point is that, before filtering, with $\lambda$ an $\mathcal{F}_{0}$-measurable random variable (and hence a known parameter), then advance knowledge of $\xi_{T}=B_{T}+\lambda T$ is indistinguishable from advance knowledge of $B_{T}$. We will see that the two cases become distinct as soon as we move to a filtration under which $\lambda$ is an unknown parameter, and we have to resort to filtering methods to find the best estimate of the stock price drift given price observations and the inside information.

Further, for $a=1$, in which case $\mathbb{F}^{L}=\mathbb{F}^{S_{T}}$, the decomposition (53) becomes

$$
B_{t}=B_{t}^{S_{T}}+\int_{0}^{t}\left(\frac{\xi_{T}-\xi_{s}}{T-s}-\lambda\right) d s .
$$

Using this in the stock price $\operatorname{SDE}(21)$, we may write the dynamics of $S$ with respect to $\mathbb{F}^{S_{T}}$ as

$$
\frac{d S_{t}}{\sigma S_{t}}=\left(d B^{S_{T}}+\frac{\xi_{T}-\xi_{t}}{T-t} d t\right) .
$$

Recall the observation filtration of the insider for $a=1, \widehat{\mathbb{F}}^{S_{T}}$. The left hand side of (55) is manifestly $\widehat{\mathbb{F}}^{S_{T}}$-adapted, as is the finite variation term on the right-hand side. This suggests (and we will prove this later) that the $\mathbb{F}^{S_{T}}$-Brownian motion $B^{S_{T}}$ is also an $\widehat{\mathbb{F}}^{S_{T}}$-Brownian motion, so we have $B^{S_{T}}=\widehat{B}^{S_{T}}$. Then (55) immediately gives the stock price dynamics under the insider's observation filtration, and any filtering is rendered redundant. The salient point is that by having knowledge at time zero of both the initial and final stock prices, the insider has already obtained, at time zero, her best estimate of the risk premium $\lambda$. This will ultimately lead to an explosion in expected utility when the insider has precise knowledge of the final stock price.

\subsection{Linear filtering with initial enlargement}

Having enlarged the filtration $\mathbb{F}$ by $\sigma(L)$ to obtain $\mathbb{F}^{L}$, we write the stock price dynamics with respect to the enlarged filtration $\mathbb{F}^{L}$. The resulting risk premium $\lambda^{L}$ is an $\mathbb{F}^{L}$-adapted process which we treat as an unobservable signal process in a filtering framework. The filtering algorithm, presented below, estimates $\lambda^{L}$ based on stock price observations augmented with the information provided at time zero by knowledge of the value of $L$. It turns out that the usual form of the Kalman-Bucy filter equations hold in this case, with initial conditions reflecting the inside information, provided we have first written the dynamics of the signal and observations processes with respect to the enlarged filtration $\mathbb{F}^{L}$. Technical integrability conditions, needed for the applicability of the Kalman filter, mean that this procedure is only valid over $[0, T]$ for $a<1$ (corresponding to strictly noisy inside information), or if $a=1$, then the algorithm is only valid up to a time $T^{*}<T$, as will be seen shortly. The rather singular case corresponding to exact information on $B_{T}$ or $S_{T}$ will require some separate reasoning to obtain the optimal investment results.

In both cases of inside information, that is $L=L_{B}$ or $L=L_{S}$, the information drift (as a function of the Brownian motion $B$, though not as a function of $L$ ) is the same, given by (50) and (54), so we write $\nu^{L} \equiv \nu^{B}=\nu^{S}$ below. The following argument then applies to both choices for $L$ (recall that with $\lambda$ being $\mathcal{F}_{0}$-measurable, $B_{T}$ and $\xi_{T}=B_{T}+\lambda T$ represent, at this stage, the same inside information).

The semimartingale decomposition (49) or (53) may then be written in unified form as

$$
B_{t}=B_{t}^{L}+\int_{0}^{t} \nu_{s}^{L} d s, \quad 0 \leq t \leq T,
$$


where $B^{L}$ is an $\mathbb{F}^{L}$-Brownian motion and with $\nu^{L}$ given by

$$
\nu_{t}^{L}=\frac{a\left(B_{T}-B_{t}\right)+(1-a) \epsilon}{a\left(T_{a}-t\right)}, \quad 0 \leq t \leq T .
$$

Using the decomposition (56) along with the stock price dynamics (21) we find that, with respect to $\mathbb{F}^{L}$, the stock price SDE is

$$
d S_{t}=\sigma S_{t}\left(\lambda_{t}^{L} d t+d B_{t}^{L}\right),
$$

where $\lambda^{L}$ is the $\mathbb{F}^{L}$-adapted process given by

$$
\lambda_{t}^{L}:=\lambda+\nu_{t}^{L}=\lambda+\frac{a\left(B_{T}-B_{t}\right)+(1-a) \epsilon}{a\left(T_{a}-t\right)}, \quad 0 \leq t \leq T .
$$

We apply the Itô formula to $\lambda^{L}$ under $\mathbb{F}^{L}$, so $\lambda$ and $B_{T}$ are treated as constants, and $B$ is an $\mathbb{F}^{L}$-semimartingale with decomposition (56). This gives, with respect to $\mathbb{F}^{L}$, the $\mathrm{SDE}$

$$
d \lambda_{t}^{L}=-\frac{1}{T_{a}-t} d B_{t}^{L}, \quad \lambda_{0}^{L}=\lambda+\frac{a B_{T}+(1-a) \epsilon}{a T_{a}} .
$$

The returns process $\xi$ in (22) has dynamics given by $d \xi_{t}=d S_{t} /\left(\sigma S_{t}\right)$, so using (57), we have dynamics with respect to $\mathbb{F}^{L}$ given by

$$
d \xi_{t}=\lambda_{t}^{L} d t+d B_{t}^{L}, \quad \xi_{0}=0 .
$$

We wish to consider an insider who trades the stock and who has access to the filtration $\widehat{\mathbb{F}}^{L}$ defined in (24). Since $\lambda$ is an unknown constant, then $\lambda^{L}$ is an unobservable signal process with $\mathbb{F}^{L}$-dynamics (58), and $\xi$ is an observation process with $\mathbb{F}^{L}$-dynamics (59). We wish to apply a filter to compute the conditional expectation and variance

$$
\widehat{\lambda}_{t}^{L}:=E\left[\lambda_{t}^{L} \mid \widehat{\mathcal{F}}_{t}^{L}\right], \quad V_{t}^{L}:=E\left[\left(\lambda_{t}^{L}-\widehat{\lambda}_{t}^{L}\right)^{2} \mid \widehat{\mathcal{F}}_{t}^{L}\right], \quad 0 \leq t \leq T .
$$

To apply the filter we also need the prior distribution of $\lambda^{L}$ given $\widehat{\mathcal{F}}_{0}^{L}$. This will be deduced from the prior distribution of $\lambda$ given $\widehat{\mathcal{F}}_{0}$ in Assumption 1, and will turn out to be Gaussian, given by (29). Then we have all the ingredients for a variant of the Kalman-Bucy filter to hold. The difference between the usual Kalman-Bucy filter and the situation here is that the observation process $\xi$ does not, on its own, constitute the entire information on which we estimate the signal. The observations are augmented with the information provided by knowledge of the value of $L$. This is absorbed into the prior distribution (29), and into the dynamics of the signal and observations by writing these with respect to $\mathbb{F}^{L}$. The result is that the following proposition looks virtually identical to the Kalman-Bucy filter. The proof follows exactly the same lines as the conventional innovations-based proof of the usual Kalman-Bucy filter, so we only give the outline.

Proposition 3. On a probability space $(\Omega, \mathcal{F}, P)$ equipped with a filtration $\mathbb{F}=\left(\mathcal{F}_{t}\right)_{0 \leq t \leq T}$ and an $\mathcal{F}$-measurable random variable $L$, define the enlarged filtration $\mathbb{F}^{L}=\left(\mathcal{F}_{t}^{L}\right)_{0 \leq t \leq T}$ by

$$
\mathcal{F}_{t}^{L}:=\mathcal{F}_{t} \vee \sigma(L), \quad 0 \leq t \leq T .
$$

Let $\lambda^{L}=\left(\lambda_{t}^{L}\right)_{0 \leq t \leq T}$ be an $\mathbb{F}^{L}$-adapted signal process satisfying

$$
d \lambda_{t}^{L}=-\frac{1}{T_{a}-t} d B_{t}^{L},
$$

where $B^{L}$ is an $\mathbb{F}^{L}$-Brownian motion and $T_{a}>T$. Let $\xi=\left(\xi_{t}\right)_{0 \leq t \leq T}$ be an $\mathbb{F}^{L}$-adapted observation process satisfying

$$
d \xi_{t}=\lambda_{t}^{L} d t+d B_{t}^{L}, \quad \xi_{0}=0
$$

and let $\widehat{\mathbb{F}}=\left(\widehat{\mathcal{F}}_{t}\right)_{0 \leq t \leq t}$ be the filtration generated by $\xi$. Define the filtration $\widehat{\mathbb{F}}^{L}=\left(\widehat{\mathcal{F}}_{t}^{L}\right)_{0 \leq t \leq T}$ by

$$
\widehat{\mathcal{F}}_{t}^{L}:=\widehat{\mathcal{F}}_{t} \vee \sigma(L) .
$$


Suppose $\lambda_{0}^{L}$ is an $\mathcal{F}_{0}^{L}$-measurable random variable with distribution given $\widehat{\mathcal{F}}_{0}^{L}$ that is Gaussian with mean $m_{L}$ and variance $\Sigma_{L}$, independent of $B^{L}$. Then the conditional expectation

$$
\widehat{\lambda}_{t}^{L}:=E\left[\lambda_{t}^{L} \mid \widehat{\mathcal{F}}_{t}^{L}\right], \quad 0 \leq t \leq T,
$$

satisfies

$$
d \widehat{\lambda}_{t}^{L}=\left(V_{t}^{L}-\frac{1}{T_{a}-t}\right) d \widehat{B}_{t}^{L}, \quad \widehat{\lambda}_{0}^{L}=m_{L},
$$

where $\widehat{B}^{L}$ is an $\widehat{\mathbb{F}}^{L}$-Brownian motion, the innovations process, satisfying

$$
d \widehat{B}_{t}^{L}=d \xi_{t}-\widehat{\lambda}_{t}^{L} d t
$$

and $V^{L}$ is the conditional variance of $\lambda^{L}$, defined by

$$
V_{t}^{L}:=E\left[\left(\lambda_{t}^{L}-\widehat{\lambda}_{t}^{L}\right)^{2} \mid \widehat{\mathcal{F}}_{t}\right], \quad 0 \leq t \leq T
$$

which is independent of $\widehat{\mathbb{F}}^{L}$ and satisfies the ordinary differential equation

$$
\frac{d V^{L}}{d t}=\frac{2}{T_{a}-t} V_{t}^{L}-\left(V_{t}^{L}\right)^{2}, \quad V_{0}^{L}=\Sigma_{L}
$$

Proof. With the inequality $T_{a}>T$, we have the integrability condition

$$
\int_{0}^{T}\left(\frac{1}{T_{a}-t}\right)^{2} d t<\infty
$$

This ensures that the conditions of Theorem 10.3 in Liptser and Shiryaev [23] are satisfied, with the proviso that the initial observation filtration has been enlarged by $\sigma(L)$. But this does not alter the mechanics of the proof of the filtering equations provided we have written the dynamics of the signal $\lambda^{L}$ and the observations $\xi$ with respect to the enlarged filtration $\mathbb{F}^{L}$. Then the filtering algorithm does indeed estimate the signal based on the enlarged observation filtration $\widehat{\mathbb{F}}^{L}$.

Direct application of Theorem 10.3 in [23] gives that with respect to $\widehat{\mathbb{F}}^{L}$ the Gaussian process $\left(\widehat{\lambda}_{t}^{L}, \xi_{t}\right)$ satisfies the SDEs $(62)$ and $(63)$ on $[0, T]$, where $\widehat{B}^{L}$ is $\widehat{\mathbb{F}}^{L}$-Brownian motion and the conditional variance $V^{L}$ satisfies the ordinary differential equation (65).

Remark 1. In the proof of Proposition 3, the condition $T_{a}>T$ is used to ensure that we have the integrability condition (66), so that Theorem 10.3 of [23] is applicable. by

If, instead, we have $T_{a} \geq T$, corresponding to $0<a \leq 1$ in (26) or (27), then (66) is replaced

$$
\int_{0}^{T^{*}}\left(\frac{1}{T_{a}-t}\right)^{2} d t<\infty
$$

for any $T^{*}<T$, and then application of Theorem 10.3 in [23] would yield that, with respect to $\widehat{\mathbb{F}}^{L}, \xi$ satisfies the SDE (63) over $[0, T)$, and $\widehat{\lambda}^{L}, V^{L}$ then satisfy (62) and (65) over [0,T).

\section{Proofs of the main theorems}

Proof of Theorem 2. The inside information is knowledge at time zero of the random variable $L=L_{B}$ given by (26). We enlarge the filtration $\mathbb{F}$ by $\sigma\left(L_{B}\right)$. With respect to the enlarged filtration $\mathbb{F}^{L}=\mathbb{F}^{L_{B}} \equiv \mathbb{F}^{B}$ the semimartingale decomposition of the Brownian motion $B$ is obtained from Example 1 and given by

$$
B_{t}=B_{t}^{B}+\int_{0}^{t} \nu_{s}^{B} d s, \quad 0 \leq t \leq T
$$


where the information drift $\nu^{L_{B}} \equiv \nu^{B}$ is given by (50) and $B^{L_{B}} \equiv B^{B}$ is an $\mathbb{F}^{B}$-Brownian motion. Using this decomposition and the stock price dynamics (21), we write the stock price SDE with respect to $\mathbb{F}^{B}$ as in (57), with $L=L_{B}$, to give

$$
d S_{t}=\sigma S_{t}\left(\lambda_{t}^{B} d t+d B_{t}^{B}\right)
$$

where the $\mathbb{F}^{B}$-adapted risk premium $\lambda^{B} \equiv \lambda^{L_{B}}$ is given by

$$
\lambda_{t}^{B}:=\lambda+\nu_{t}^{B}=\lambda+\frac{L_{B}-a B_{t}}{a\left(T_{a}-t\right)}=\lambda+\frac{a\left(B_{T}-B_{t}\right)+(1-a) \epsilon}{a\left(T_{a}-t\right)}, \quad 0 \leq t \leq T .
$$

We apply the Itô formula to $\lambda^{B}$ under $\mathbb{F}^{B}$ and use the semi-martingale decomposition (67) (so that $\lambda$ and $B_{T}$ are treated as known constants) to obtain (58) with $L=L_{B}$, as

$$
d \lambda_{t}^{B}=-\frac{1}{T_{a}-t} d B_{t}^{B}, \quad \lambda_{0}^{B}=\lambda+\frac{L_{B}}{a T_{a}} .
$$

The returns process $\xi$ in (22) satisfies $d \xi_{t}=d S_{t} /\left(\sigma S_{t}\right)$, so from (68) its dynamics with respect to $\mathbb{F}^{B}$ are given by

$$
d \xi_{t}=\lambda_{t}^{B} d t+d B_{t}^{B}
$$

Now consider the system (69) and (70) as a linear signal and observation system in a filtering framework. To this end, define the conditional expectation and variance in (60) with $L=L_{B}$ :

$$
\widehat{\lambda}_{t}^{B}:=E\left[\lambda_{t}^{B} \mid \widehat{\mathcal{F}}_{t}^{B}\right], \quad V_{t}^{B}:=E\left[\left(\lambda_{t}^{B}-\widehat{\lambda}_{t}^{B}\right)^{2} \mid \widehat{\mathcal{F}}_{t}^{B}\right], \quad 0 \leq t \leq T,
$$

where $\widehat{\mathbb{F}}^{B}=\left(\widehat{\mathcal{F}}_{t}^{B}\right)_{0 \leq t \leq T}$ is the enlarged observation filtration, defined by (24) with $L=L_{B}$ :

$$
\widehat{\mathcal{F}}_{t}^{B}:=\widehat{\mathcal{F}}_{t} \vee \sigma\left(L_{B}\right), \quad 0 \leq t \leq T,
$$

and we have written $\widehat{\lambda}^{L_{B}} \equiv \widehat{\lambda}^{B}, V^{L_{B}} \equiv V^{B}$ for brevity of notation.

To apply our variant of the Kalman-Bucy filter, Proposition 3, we also need the prior distribution of $\lambda_{0}^{B}$ given $\widehat{\mathcal{F}}_{0}^{B}$. The prior distribution distribution of $\lambda$ is given in Assumption 1. Since $B_{T}$ and $\epsilon$ in (26) are independent of each other and of $\lambda$, then $L_{B}$ is also independent of $\lambda$. Hence the distribution of $\lambda$ given $\widehat{\mathcal{F}}_{0}^{B}$ is the same as its distribution given $\widehat{\mathcal{F}}_{0}$, and using the initial condition in (69), the probability law for $\lambda_{0}^{B}$ given $\widehat{\mathcal{F}}_{0}^{B}$ is therefore

$$
\operatorname{Law}\left(\lambda_{0}^{B} \mid \widehat{\mathcal{F}}_{0}^{B}\right)=\operatorname{Law}\left(\lambda+\frac{L_{B}}{a T_{a}} \mid \widehat{\mathcal{F}}_{0}^{B}\right)=\mathrm{N}\left(\lambda_{0}+\frac{L_{B}}{a T_{a}}, \mathrm{v}_{0}\right), \quad \text { independent of } B^{B} .
$$

Of course, since $L_{B}$ is $\widehat{\mathcal{F}}_{0}^{B}$-measurable, it acts as a constant in the above computation, and since $\lambda$ is independent of $B$ and $L_{B}$, it is independent of $B^{B}$, and so $\lambda_{0}^{B}$ is also independent of $B^{B}$.

Now assume $a \neq 1$. We apply the filtering algorithm in Proposition 3 with $L=L_{B}$ to obtain that $\widehat{\lambda}^{B}$ satisfies the analogue of (62) with $L=L_{B}$, over the timeframe $[0, T]$ :

$$
d \widehat{\lambda}_{t}^{B}=\left(V_{t}^{B}-\frac{1}{T_{a}-t}\right) d \widehat{B}_{t}^{B}, \quad \widehat{\lambda}_{0}^{B}=\lambda_{0}+\frac{L_{B}}{a T_{a}},
$$

where $\widehat{B}^{B} \equiv \widehat{B}^{L_{B}}$ is an $\widehat{\mathbb{F}}^{B}$-Brownian motion, the innovations process, satisfying

$$
d \widehat{B}_{t}^{B}=d \xi_{t}-\widehat{\lambda}_{t}^{B} d t
$$

and the conditional variance $V^{B} \equiv V^{L_{B}}$ satisfies the analogue of (65) with $L=L_{B}$ :

$$
\frac{d V^{B}}{d t}=\frac{2}{T_{a}-t} V_{t}^{B}-\left(V_{t}^{B}\right)^{2}, \quad V_{0}^{B}=\mathrm{v}_{0} .
$$

Now define $\mathrm{v}^{B} \equiv \mathrm{v}^{L_{B}}$ by

$$
\mathrm{v}_{t}^{B}:=V_{t}^{B}-\frac{1}{T_{a}-t}, \quad 0 \leq t \leq T .
$$


Then $\mathrm{v}^{B}$ satisfies a Riccati equation of the same form as (3):

$$
\frac{d \mathrm{v}_{t}^{B}}{d t}=-\left(\mathrm{v}_{t}^{B}\right)^{2}, \quad \mathrm{v}_{0}^{B}=\mathrm{v}_{0}-\frac{1}{T_{a}},
$$

which has solution as given in (42). We also have $V^{B} \geq 0$ for all $t \in[0, T]$, which follows from direct calculation and $\mathrm{v}_{0}>0$.

The SDE (72) for $\widehat{\lambda}^{B} \equiv \widehat{\lambda}^{L_{B}}$ then becomes

$$
d \widehat{\lambda}_{t}^{B}=\mathrm{v}_{t}^{B} d \widehat{B}_{t}^{B}=\mathrm{v}_{t}^{B}\left(d \xi_{t}-\widehat{\lambda}_{t}^{B} d t\right), \quad \widehat{\lambda}_{0}^{B}=\lambda_{0}+\frac{L_{B}}{a T_{a}}
$$

where we have used (73). Then the solution for $\widehat{\lambda}^{B}$ is

$$
\widehat{\lambda}_{t}^{B}=\widehat{\lambda}_{0}^{B}+\int_{0}^{t} \mathrm{v}_{s}^{B} d \widehat{B}_{s}^{B}, \quad 0 \leq t \leq T,
$$

or, in terms of $\xi$, as given in equation (42) of the theorem.

Using (73), the stock price $\operatorname{SDE} d S_{t}=\sigma S_{t} d \xi_{t}$ with respect to $\widehat{\mathbb{F}}^{B}$ becomes

$$
d S_{t}=\sigma S_{t}\left(\widehat{\lambda}_{t}^{B} d t+d \widehat{B}_{t}^{B}\right)
$$

and the insider is investing in a stock following these dynamics using an $\widehat{\mathbb{F}}^{B}$-adapted strategy $\theta^{B}$.

From (74) and (75) we have $E\left[\int_{0}^{T}\left(\widehat{\lambda}_{t}^{B}\right)^{2} d t \mid \widehat{\mathcal{F}}_{0}^{L}\right]<\infty$, a.s. From (74)-(76) we see that we have recovered a model of the same form as that of Section 2, and the solution to the optimal investment problem for $a<1$ then follows from Theorem 1 with $\left(\widehat{\mathbb{F}}^{B}, \widehat{B}^{B}, \widehat{\lambda}^{B}, \mathrm{v}^{B}\right)$ in place of $(\mathbb{F}, B, \delta, \mathrm{w})$, and the condition (41) on $\mathrm{v}_{0}$ given in the theorem for $0<p<1$ ensures that the solution is well defined.

Now suppose $a=1$ and $\mathrm{v}_{0}>0$. Then $L=B_{T}$ and $T_{a}=T$, and the initial value of the effective variance $\mathrm{v}^{B_{T}}$ is given by

$$
\mathrm{v}_{0}^{B_{T}}=\mathrm{v}_{0}-\frac{1}{T}
$$

Moreover, the integrability condition (66) fails to hold and we can only apply the filtering algorithm up to some terminal time $T^{*}<T$, in accordance with Remark 1 . Then the arguments leading to $(74)-(76)$ are applicable over the time interval $[0, T)$.

To establish the results for $t=T$, define

$$
\widehat{\lambda}_{T}^{B_{T}}:=\lim _{t \rightarrow T} E\left[\lambda_{t}^{B_{T}} \mid \widehat{\mathcal{F}}_{t}^{B_{T}}\right]=\lim _{t \rightarrow T} \widehat{\lambda}_{t}^{B_{T}}=\lim _{t \rightarrow T} \frac{\widehat{\lambda}_{0}^{B_{T}}+\mathrm{v}_{0}^{B_{T}} \xi_{t}}{1+\mathrm{v}_{0}^{B_{T}} t}=\frac{\widehat{\lambda}_{0}^{B_{T}}+\mathrm{v}_{0}^{B_{T}} \xi_{T}}{1+\mathrm{v}_{0}^{B_{T}} T}
$$

which is well defined since $\xi_{t}$ is continuous on $[0, T]$, and $1+\mathrm{v}_{0}^{B_{T}} T=\mathrm{v}_{0} T \neq 0$. Since $\widehat{B}_{t}^{B_{T}}$ is defined only for $t \in[0, T)$ we need to define $\widehat{B}_{T}^{B_{T}}$. To this end, define

$$
\widehat{B}_{T}^{B_{T}}:=\lim _{t \rightarrow T} \widehat{B}_{t}^{B_{T}}=\lim _{t \rightarrow T}\left[\xi_{t}-\int_{0}^{t} \widehat{\lambda}_{s}^{B_{T}} d s\right]
$$

which is well defined due to continuity of $\xi_{t}$ and $\hat{\lambda}_{t}^{B}$ on $[0, T]$ (the latter due to (77)). From this it follows that the relation

$$
\xi_{t}=\int_{0}^{t} \widehat{\lambda}_{s}^{B_{T}} d s+\widehat{B}_{t}^{B_{T}}, \quad 0 \leq t \leq T
$$

as well as (75), both hold over $[0, T]$. This, along with the Itô formula yields that $\widehat{\lambda}^{B_{T}}$ is given by

$$
\widehat{\lambda}_{t}^{B_{T}}=\lambda_{0}+\frac{B_{T}}{T}+\int_{0}^{t} \mathrm{v}_{s}^{B_{T}} d \widehat{B}_{s}^{B_{T}}=\frac{\widehat{\lambda}^{B_{T}}+\mathrm{v}_{0}^{B_{T}} \xi_{t}}{1+\mathrm{v}_{0}^{B_{T}} t}, \quad 0 \leq t \leq T,
$$


with $\mathrm{v}^{B_{T}}$ given by

$$
\mathrm{v}_{t}^{B_{T}}=\frac{\mathrm{v}_{0}^{B_{T}}}{1+\mathrm{v}_{0}^{B_{T}} t}, \quad 0 \leq t \leq T,
$$

for $t \in[0, T]$. This establishes that $(74)-(76)$ do in fact hold over the entire time interval $[0, T]$ and the rest of the proof of the optimal investment result goes through unaltered.

Finally, for the case $a=1$ and $\mathrm{v}_{0}=0$, we require a different argument, similar to the one in Pikovsky and Karatzas [27], which considers the optimal investment problem over some subinterval $\left[0, T^{*}\right]$, for some $T^{*}<T$. Indeed, note that when $\mathrm{v}_{0}=0$ there is no uncertainty in the risk premium $\lambda$ and we are back in the scenario considered by [27]. The risk premium $\lambda$ is equal to the constant $\lambda_{0}$ and the returns process $\xi$ in (22) satisfies

$$
\xi_{t}=\lambda_{0} t+B_{t}, \quad 0 \leq t \leq T .
$$

This implies that $\widehat{\mathbb{F}}=\mathbb{F}$ and also $\widehat{\mathbb{F}}^{B_{T}}=\mathbb{F}^{B_{T}}$. The decomposition of the Brownian motion $B$ under $\mathbb{F}^{B_{T}}$ is given by the Brownian bridge relation (51). Then, with respect to $\mathbb{F}^{B_{T}}$ the stock price dynamics (21) are therefore given by

$$
d S_{t}=\sigma S_{t}\left(\lambda_{t}^{B_{T}} d t+d B_{t}^{B_{T}}\right),
$$

where the $\mathbb{F}^{B_{T}}$-adapted risk premium process $\lambda^{B_{T}}$ is given by

$$
\lambda_{t}^{B_{T}}=\lambda_{0}+\frac{B_{T}-B_{t}}{T-t}, \quad 0 \leq t \leq T,
$$

and we have used the fact that $\lambda=\lambda_{0}$ for $\mathrm{v}_{0}=0$. For any $T^{*}<T$ we have

$$
E\left[\int_{0}^{T^{*}}\left(\lambda_{t}^{B_{T}}\right)^{2} d t \mid \mathcal{F}_{0}^{B_{T}}\right]<\infty, \text { a.s. }
$$

Note also that we have the dynamics (with respect to $\mathbb{F}^{B_{T}}$ ) for $\lambda^{B_{T}}$ :

$$
d \lambda_{t}^{B_{T}}=-\frac{1}{T-t} d B_{t}^{B_{T}} .
$$

In this case define the effective variance $\mathrm{v}^{B_{T}}$ by

$$
\mathrm{v}_{t}^{B_{T}}:=-\frac{1}{T-t}, \quad 0 \leq t \leq T^{*}<T,
$$

and we then have

$$
\mathrm{v}_{t}^{B_{T}}=\frac{\mathrm{v}_{0}^{B_{T}}}{1+\mathrm{v}_{0}^{B_{T}} t}, \quad 0 \leq t \leq T^{*}<T
$$

as well as

$$
\lambda_{t}^{B_{T}}=\lambda_{0}^{B_{T}}+\int_{0}^{t} \mathrm{v}_{s}^{B_{T}} d B_{s}^{B_{T}}, \quad 0 \leq t \leq T^{*}<T .
$$

In other words we still have an optimal investment model of the form in Section 2 over the sub-interval $\left[0, T^{*}\right]$, for any $T^{*}<T$.

We introduce a sequence of auxiliary optimal investment problems over a sub-interval $\left[0, T^{n}\right]$, for $T^{n}:=T-1 / n$ and $n \in \mathbb{N}$. Define the value function

$$
u_{B_{T}}\left(x ; T^{n}, \mathbb{F}^{B_{T}}\right):=\sup _{\theta^{B_{T}} \in \mathcal{A}\left(T^{n} ; \mathbb{F}^{B_{T}}\right)} E\left[U_{p}\left(X_{T^{n}}^{B_{T}}\right) \mid \mathcal{F}_{0}^{B_{T}}\right] .
$$

which is the maximum expected utility over the class $\mathcal{A}\left(T^{n} ; \mathbb{F}^{B_{T}}\right)$ of $\mathbb{F}^{B_{T}}$-adapted portfolios on the subinterval $\left[0, T^{n}\right]$. We are within the framework of the classical theory covered by Theorem 1 , and the value of the maximum utility for the problem in (78) is therefore easily read off from 
Theorem 1 with $\left(\mathbb{F}^{B_{T}}, B^{B_{T}}, \lambda^{B_{T}}, \mathrm{v}^{B_{T}}\right)$ in place of $(\mathbb{F}, B, \delta, \mathrm{w})$, and with $T^{n}$ in place of $T$. We then have

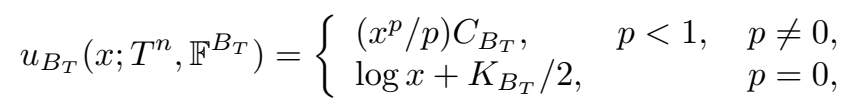

where $C_{B_{T}}, K_{B_{T}}$ are $\mathcal{F}_{0}^{B_{T}}$-measurable random variables given by

$$
\begin{aligned}
C_{B_{T}} & =\left[\left(1-T^{n} / T\right)^{p}\left(1-q T^{n} / T\right)^{1-p}\right]^{-1 / 2} \exp \left(-\frac{q\left(\lambda_{0}^{B_{T}}\right)^{2} T^{n}}{2\left(1-q T^{n} / T\right)}\right), \frac{1}{p}+\frac{1}{q}=1, \\
K_{B_{T}} & =\left(\left(\lambda_{0}^{B_{T}}\right)^{2}-1 / T\right) T^{n}-\log \left(1-T^{n} / T\right),
\end{aligned}
$$

and where we have used the explicit form for $\mathrm{v}_{0}^{B_{T}}$. The optimal $\mathbb{F}^{B_{T}}$-adapted trading strategy achieving the supremum in (78) is $\theta^{B_{T}, *}$, given by

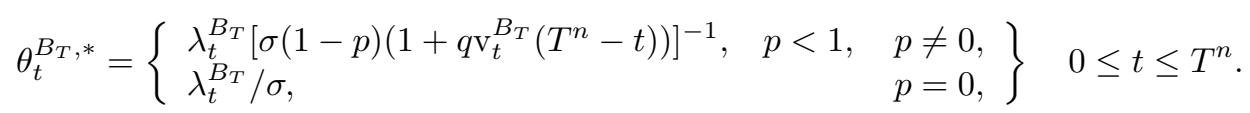

To conclude that the value function of the insider is as claimed, consider the following strategy in $\mathcal{A}\left(T ; \mathbb{F}^{B}\right)$. Define a portfolio $\theta^{B_{T}, n}$, for any $T^{n}<T$, by

$$
\theta_{t}^{B_{T}, n}:=\theta_{t}^{B_{T}, *} \mathbb{1}_{\left\{t \leq T^{n}\right\}}, \quad 0 \leq t \leq T,
$$

which corresponds to the strategy of investing optimally up to $T^{n}$ then moving all the investments to the risk-free asset and keeping them there until time $T$. Since this strategy is admissible for the original problem over the interval $[0, T]$, we have that

$$
u_{B_{T}}\left(x ; T^{n}, \mathbb{F}^{B_{T}}\right) \leq u_{B_{T}}\left(x ; T, \mathbb{F}^{B_{T}}\right) .
$$

Therefore, taking limits in (79) yields

$$
u_{B_{T}}\left(x ; T, \mathbb{F}^{B_{T}}\right) \geq \lim _{n \rightarrow+\infty} u_{B_{T}}\left(x ; T^{n}, \mathbb{F}^{B_{T}}\right)= \begin{cases}\infty, & p \in[0,1), \\ 0, & p<0,\end{cases}
$$

Since $u_{B_{T}}(x) \leq+\infty$ for $p \in(0,1)$ and $u_{B_{T}}(x) \leq 0$ for $p \in(-\infty, 0)$, the result follows.

Proof of Theorem 3. The inside information is knowledge at time zero of the random variable $L=L_{S}$ given by $(27)$. We enlarge the filtration $\mathbb{F}$ by $\sigma\left(L_{S}\right)$. With respect to the enlarged filtration $\mathbb{F}^{L}=\mathbb{F}^{L_{S}} \equiv \mathbb{F}^{S}$ the semimartingale decomposition of the Brownian motion $B$ is obtained from Example 2 and given by

$$
B_{t}=B_{t}^{S}+\int_{0}^{t} \nu_{s}^{S} d s, \quad 0 \leq t \leq T,
$$

where the information drift $\nu^{L_{S}} \equiv \nu^{S}$ is given by (54) and $B^{L_{S}} \equiv B^{S}$ is an $\mathbb{F}^{S}$-Brownian motion. Using this decomposition and the stock price dynamics (21), we write the stock price SDE with respect to $\mathbb{F}^{S}$ as in (57), with $L=L_{S}$, to give

$$
d S_{t}=\sigma S_{t}\left(\lambda_{t}^{S} d t+d B_{t}^{S}\right),
$$

where the $\mathbb{F}^{S}$-adapted risk premium $\lambda^{S} \equiv \lambda^{L_{S}}$ is given by

$$
\lambda_{t}^{S}:=\lambda+\nu_{t}^{S}=\lambda+\frac{L_{S}-a\left(B_{t}+\lambda T\right)}{a\left(T_{a}-t\right)}=\lambda+\frac{a\left(B_{T}-B_{t}\right)+(1-a) \epsilon}{a\left(T_{a}-t\right)}, \quad 0 \leq t \leq T .
$$

Using (22) we can also write the information drift in terms of $\xi$ and $\lambda$ :

$$
\nu_{t}^{S}=\frac{L_{S}-a \xi_{t}}{a\left(T_{a}-t\right)}-\lambda\left(\frac{T-t}{T_{a}-t}\right),
$$


which is more natural when we are considering inside information based on knowledge of $\xi_{T}$.

We apply the Itô formula to $\lambda^{S}$ under $\mathbb{F}^{S}$ and use the semi-martingale decomposition (81) to obtain (58) with $L=L_{S}$, as

$$
d \lambda_{t}^{S}=-\frac{1}{T_{a}-t} d B_{t}^{S}, \quad \lambda_{0}^{S}=\lambda\left(1-\frac{T}{T_{a}}\right)+\frac{L_{S}}{a T_{a}} .
$$

The returns process $\xi$ in (22) satisfies $d \xi_{t}=d S_{t} /\left(\sigma S_{t}\right)$, so from (82) its dynamics with respect to $\mathbb{F}^{S}$ are given by

$$
d \xi_{t}=\lambda_{t}^{S} d t+d B_{t}^{S} .
$$

Now consider the system (84) and (85) as a linear signal and observation system in a filtering framework. To this end, define the conditional expectation and variance in (60) with $L=L_{S}$ :

$$
\widehat{\lambda}_{t}^{S}:=E\left[\lambda_{t}^{S} \mid \widehat{\mathcal{F}}_{t}^{S}\right], \quad V_{t}^{S}:=E\left[\left(\lambda_{t}^{S}-\widehat{\lambda}_{t}^{S}\right)^{2} \mid \widehat{\mathcal{F}}_{t}^{S}\right], \quad 0 \leq t \leq T,
$$

where $\widehat{\mathbb{F}}^{S}=\left(\widehat{\mathcal{F}}_{t}^{S}\right)_{0 \leq t \leq T}$ is the enlarged observation filtration, defined by (24) with $L=L_{S}$ :

$$
\widehat{\mathcal{F}}_{t}^{S}:=\widehat{\mathcal{F}}_{t} \vee \sigma\left(L_{S}\right), \quad 0 \leq t \leq T,
$$

and we have written $\widehat{\lambda}^{L_{S}} \equiv \widehat{\lambda}^{S}, V^{L_{S}} \equiv V^{S}$ for brevity of notation.

To apply our variant of the Kalman-Bucy filter, Proposition 3, we also need the prior distribution of $\lambda_{0}^{S}$ given $\widehat{\mathcal{F}}_{0}^{S}$. The prior distribution distribution of $\lambda$ is given in Assumption 1. However, the distribution of $\lambda$ conditional on $\widehat{\mathcal{F}}_{0}^{S}$ is altered from that in Assumption 1 because the inside information (related as it is to $S_{T}$ ) contributes to the estimation of $\lambda$. We have the following lemma.

Lemma 2. Conditional on $\widehat{\mathcal{F}}_{0}^{S}, \lambda$ is Gaussian, with

$$
E\left[\lambda \mid \widehat{\mathcal{F}}_{0}^{S}\right]=\frac{\lambda_{0}+\mathrm{v}_{0} T\left(L_{S} / a T_{a}\right)}{1+\mathrm{v}_{0} T\left(T / T_{a}\right)}, \quad \operatorname{var}\left[\lambda \mid \widehat{\mathcal{F}}_{0}^{S}\right]=\frac{\mathrm{v}_{0}}{1+\mathrm{v}_{0} T\left(T / T_{a}\right)} .
$$

Proof. For two independent Gaussian random variables $X$ and $Y$ distributed according to $\mathrm{N}\left(\mu_{X}, \sigma_{X}^{2}\right)$ and $\mathrm{N}\left(\mu_{Y}, \sigma_{Y}^{2}\right)$ respectively, we have

$$
E[X \mid X+Y]=\mu_{X}+\frac{\sigma_{X}^{2}}{\sigma_{X}^{2}+\sigma_{Y}^{2}}\left(X+Y-\mu_{X}-\mu_{Y}\right),
$$

and

$$
\operatorname{var}[X \mid X+Y]=\frac{\sigma_{X}^{2} \sigma_{Y}^{2}}{\sigma_{X}^{2}+\sigma_{Y}^{2}}
$$

We have, using $\xi_{T}=B_{T}+\lambda T$,

$$
\begin{aligned}
E\left[\lambda \mid \widehat{\mathcal{F}}_{0}^{S}\right] & =E\left[\lambda \mid L_{S}\right] \\
& =E\left[\lambda \mid a\left(B_{T}+\lambda T\right)+(1-a) \epsilon\right] \\
& =\frac{1}{a T} E\left[a \lambda T \mid a \lambda T+a B_{T}+(1-a) \epsilon\right] .
\end{aligned}
$$

Applying (88) with $X=a \lambda T \sim \mathrm{N}\left(a \lambda_{0} T, a^{2} \mathrm{v}_{0} T^{2}\right), Y=a B_{T}+(1-a) \epsilon \sim \mathrm{N}\left(0, a^{2} T_{a}\right)$, we obtain the first equality in (87). Similarly, using (89) we obtain the second equality in (87).

Using this lemma we can write down the initial distribution of $\lambda_{0}^{S}$ given $\widehat{\mathcal{F}}_{0}^{S}$. Using the formula for $\lambda_{0}^{S}$ in (84), we find that, conditional on $\widehat{\mathcal{F}}_{0}^{S}, \lambda_{0}^{S}$ is Gaussian according to $\operatorname{Law}\left(\lambda_{0}^{S} \mid \widehat{\mathcal{F}}_{0}^{S}\right)=$ $\mathrm{N}\left(\widehat{\lambda}_{0}^{S}, V_{0}^{S}\right)$, with

$$
\widehat{\lambda}_{0}^{S}=\frac{\lambda_{0}\left(1-T / T_{a}\right)+\left(1+\mathrm{v}_{0} T\right)\left(L_{S} /\left(a T_{a}\right)\right)}{1+\mathrm{v}_{0} T\left(T / T_{a}\right)}, \quad V_{0}^{S}=\frac{\left(1-\left(T / T_{a}\right)\right)^{2} \mathrm{v}_{0}}{1+\mathrm{v}_{0} T\left(T / T_{a}\right)},
$$


which defines the prior distribution of the signal process $\lambda^{S}$.

Now assume $a \neq 1$. We apply the filtering algorithm in Proposition 3 with $L=L_{S}$ to obtain that $\widehat{\lambda}^{S}$ satisfies the analogue of (62) with $L=L_{S}$, over the timeframe $[0, T]$ :

$$
d \widehat{\lambda}_{t}^{S}=\left(V_{t}^{S}-\frac{1}{T_{a}-t}\right) d \widehat{B}_{t}^{S}, \quad \widehat{\lambda}_{0}^{S}=\frac{\lambda_{0}\left(1-T / T_{a}\right)+\left(1+\mathrm{v}_{0} T\right)\left(L_{S} /\left(a T_{a}\right)\right)}{1+\mathrm{v}_{0} T\left(T / T_{a}\right)},
$$

where $\widehat{B}^{S} \equiv \widehat{B}^{L_{S}}$ is an $\widehat{\mathbb{F}}^{S}$-Brownian motion, the innovations process, satisfying

$$
d \widehat{B}_{t}^{S}=d \xi_{t}-\widehat{\lambda}_{t}^{S} d t
$$

and the conditional variance $V^{S} \equiv V^{L_{S}}$ satisfies the analogue of (65) with $L=L_{S}$ :

$$
\frac{d V^{S}}{d t}=\frac{2}{T_{a}-t} V_{t}^{S}-\left(V_{t}^{S}\right)^{2}, \quad V_{0}^{S}=\frac{\left(1-\left(T / T_{a}\right)\right)^{2} \mathrm{v}_{0}}{1+\mathrm{v}_{0} T\left(T / T_{a}\right)} .
$$

Now define $\mathrm{v}^{S} \equiv \mathrm{v}^{L_{S}}$ by

$$
\mathrm{v}_{t}^{S}:=V_{t}^{S}-\frac{1}{T_{a}-t}, \quad 0 \leq t \leq T .
$$

Then $\mathrm{v}^{S}$ satisfies a Riccati equation of the same form as (3):

$$
\frac{d \mathrm{v}_{t}^{S}}{d t}=-\left(\mathrm{v}_{t}^{S}\right)^{2}, \quad \mathrm{v}_{0}^{S}=\frac{\left(1-\left(T / T_{a}\right)\right)^{2} \mathrm{v}_{0}}{1+\mathrm{v}_{0} T\left(T / T_{a}\right)}-\frac{1}{T_{a}},
$$

which has solution as given in (44). It is easy to see that we have $V^{S} \geq 0$ for all $t \in[0, T]$.

The SDE (91) for $\widehat{\lambda}^{S} \equiv \widehat{\lambda}^{L_{S}}$ then becomes

$$
d \widehat{\lambda}_{t}^{S}=\mathrm{v}_{t}^{S} d \widehat{B}_{t}^{S}=\mathrm{v}_{t}^{S}\left(d \xi_{t}-\widehat{\lambda}_{t}^{S} d t\right), \quad \widehat{\lambda}_{0}^{S}=\frac{\lambda_{0}\left(1-T / T_{a}\right)+\left(1+\mathrm{v}_{0} T\right)\left(L_{S} /\left(a T_{a}\right)\right)}{1+\mathrm{v}_{0} T\left(T / T_{a}\right)},
$$

where we have used (92). Then the solution for $\widehat{\lambda}^{S}$ is

$$
\widehat{\lambda}_{t}^{S}=\widehat{\lambda}_{0}^{S}+\int_{0}^{t} \mathrm{v}_{s}^{S} d \widehat{B}_{s}^{S}, \quad 0 \leq t \leq T,
$$

or, in terms of $\xi$, as given in equation (44) of the theorem.

With respect to $\widehat{\mathbb{F}}^{S}$ the stock price $\mathrm{SDE} d S_{t}=\sigma S_{t} d \xi_{t}$ becomes, on using (92),

$$
d S_{t}=\sigma S_{t}\left(\widehat{\lambda}_{t}^{S} d t+d \widehat{B}_{t}^{S}\right),
$$

and the insider is investing in a stock following these dynamics using an $\widehat{\mathbb{F}}^{S}$-adapted strategy $\theta^{S}$.

From (93) and (94) we have $E\left[\int_{0}^{T}\left(\widehat{\lambda}_{t}^{S}\right)^{2} d t \mid \widehat{\mathcal{F}}_{0}^{S}\right]<\infty$ a.s. From (93)-(95) we see that we have recovered a model of the same form as that of Section 2, and the solution to the optimal investment problem for $a<1$ then follows from Theorem 1 with $\left(\widehat{\mathbb{F}}^{S}, \widehat{B}^{S}, \widehat{\lambda}^{S}, \mathrm{v}^{S}\right)$ in place of $(\mathbb{F}, B, \delta, \mathrm{w})$, and the condition (43) on $\mathrm{v}_{0}$ given in the theorem for $0<p<1$ ensures that the solution is well defined.

Now suppose $a=1$. Then from Example 2, the stock price SDE with respect to the enlarged filtration $\mathbb{F}^{S_{T}}$ is

$$
d S_{t}=\sigma S_{t}\left(\lambda_{t}^{S_{T}} d t+d B_{t}^{S_{T}}\right),
$$

where $\lambda^{S_{T}}$ is the $\mathbb{F}^{S_{T}}$-adapted process given by

$$
\lambda_{t}^{S_{T}}=\frac{\xi_{T}-\xi_{t}}{T-t}, \quad 0 \leq t \leq T .
$$

But this is also $\widehat{\mathbb{F}}^{S_{T}}$-adapted, so it immediately follows that

$$
\widehat{\lambda}_{t}^{S_{T}}=\lambda_{t}^{S_{T}}=\frac{\xi_{T}-\xi_{t}}{T-t}, \quad 0 \leq t \leq T .
$$


This suggests that we do not need any filtering procedure to estimate the risk premium $\lambda^{S_{T}}$ conditional on $\widehat{\mathbb{F}}^{S_{T}}$ when $a=1$. This is in fact the case, as we shall see shortly. Note also that, on setting $a=1$ in Lemma 2 we have

$$
E\left[\lambda \mid \widehat{\mathcal{F}}_{0}^{S_{T}}\right]=\frac{\lambda_{0}+\mathrm{v}_{0} \xi_{T}}{1+\mathrm{v}_{0} T}=\widehat{\lambda}_{T}, \quad \operatorname{var}\left[\lambda \mid \widehat{\mathcal{F}}_{0}^{S_{T}}\right]=\frac{\mathrm{v}_{0}}{1+\mathrm{v}_{0} T}=\mathrm{v}_{T} .
$$

In other words, knowing the value of the terminal stock price at time zero immediately gives the insider the best estimate of $\lambda$ that would be obtained from observing the stock price over $[0, T]$. This corresponds to the notorious result that to estimate $\lambda$ from the returns process $\xi_{t}=\lambda t+B_{t}$, using continuous observations over $[0, T]$, the only relevant observations are the initial and final ones: the best estimate of $\lambda$ from observations of $\left(\xi_{t}\right)_{0 \leq t \leq T}$ is $\bar{\lambda}(T):=\xi_{T} / T$. This is the underlying reason for the notorious difficulty of estimating the mean return of a log-Brownian stock, as discussed in Rogers [28], for example.

The returns process $\xi$ satisfies $d \xi_{t}=d S_{t} /\left(\sigma S_{t}\right)$, so from (55) its dynamics with respect to $\mathbb{F}^{S_{T}}$ are

$$
d \xi_{t}=\lambda_{t}^{S_{T}} d t+d B_{t}^{S_{T}}
$$

which implies that $B^{S_{T}}$ is $\widehat{\mathbb{F}}^{S_{T}}$-adapted, given that $\lambda^{S_{T}}=\widehat{\lambda}^{S_{T}}$ is $\widehat{\mathbb{F}}^{S_{T}}$-adapted. We now show that we have $B^{S_{T}}=\widehat{B}^{S_{T}}$, where $\widehat{B}^{S_{T}}$ is an $\widehat{\mathbb{F}}^{S_{T}}$-Brownian motion.

From (84), for $a=1$ we have dynamics for the signal process $\lambda^{S_{T}}$ given by

$$
d \lambda_{t}^{S_{T}}=-\frac{1}{T-t} d B_{t}^{S_{T}}
$$

so using Remark 1 we apply the filtering algorithm over $[0, T)$, to obtain that the SDE for $\xi$ in the observation filtration $\widehat{\mathbb{F}}^{S_{T}}$ is

$$
d \xi_{t}=\widehat{\lambda}_{t}^{S_{T}} d t+d \widehat{B}_{t}^{S_{T}}
$$

for all $t \in[0, T)$. Equating (97) and (99) gives $P\left[B_{t}^{S_{T}}=\widehat{B}_{t}^{S_{T}}, t \in[0, T)\right]=1$. Define $\widehat{B}_{T}^{S_{T}}:=$ $\limsup _{t \rightarrow T} \widehat{B}_{t}^{S_{T}}$. Then by continuity of Brownian motion we have $P\left[B_{t}^{S_{T}}=\widehat{B}_{t}^{S_{T}}, t \in[0, T]\right]=1$ and therefore the stock price follows (96) in the observation filtration, as claimed. As filtering is redundant, we drop the "hat" notation in the rest of the proof.

We are now in a similar scenario to that in the last part of the proof of Theorem 2, where we show that there is a utility explosion by using a sequence of auxiliary optimal investment problems over a sub-interval $\left[0, T^{n}\right]$, for $T^{n}:=T-1 / n$ and $n \in \mathbb{N}$. Define the value function

$$
u_{S_{T}}\left(x ; T^{n}, \mathbb{F}^{S_{T}}\right):=\sup _{\theta^{S_{T}} \in \mathcal{A}\left(T^{n} ; \mathbb{F}^{S_{T}}\right)} E\left[U_{p}\left(X_{T^{n}}^{S_{T}}\right) \mid \mathcal{F}_{0}^{S_{T}}\right] .
$$

The agent is investing in a stock with dynamics given by (96), and from the dynamics (98) of $\lambda^{S_{T}}$ we may write

$$
\lambda_{t}^{S_{T}}=\lambda_{0}^{S_{T}}+\int_{0}^{t} \mathrm{v}_{s}^{S_{T}} d B_{t}^{S_{T}}, \quad 0 \leq t \leq T^{n}
$$

where we have written

$$
\mathrm{v}_{t}^{S_{T}}=-\frac{1}{T-t}=\frac{\mathrm{v}_{0}^{S_{T}}}{1+\mathrm{v}_{0}^{S_{T}} t}, \quad 0 \leq t \leq T^{n},
$$

with $\mathrm{v}_{0}^{S_{T}}=-1 / T$. The solution to the investment problem (100) then follows from Theorem 1 with $\left(\mathbb{F}^{S_{T}}, B^{S_{T}}, \lambda^{S_{T}}, \mathrm{v}^{S_{T}}\right)$ in place of $(\mathbb{F}, B, \delta, \mathrm{w})$, and with $T^{n}$ in place of $T$. We then have

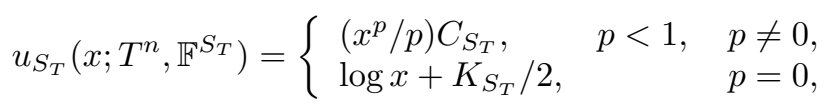

where $C_{S_{T}}, K_{S_{T}}$ are given by

$$
\begin{aligned}
C_{S_{T}} & =\left[\left(1-T^{n} / T\right)^{p}\left(1-q T^{n} / T\right)^{1-p}\right]^{-1 / 2} \exp \left(-\frac{q\left(\lambda_{0}^{S_{T}}\right)^{2} T^{n}}{2\left(1-q T^{n} / T\right)}\right), \\
K_{S_{T}} & =\left(\left(\lambda_{0}^{S_{T}}\right)^{2}-1 / T\right) T^{n}-\log \left(1-T^{n} / T\right),
\end{aligned}
$$


and where we have used the explicit form for $\mathrm{v}_{0}^{S_{T}}$. The optimal trading strategy achieving the supremum in (100) is $\theta^{S_{T}, *}$, given by

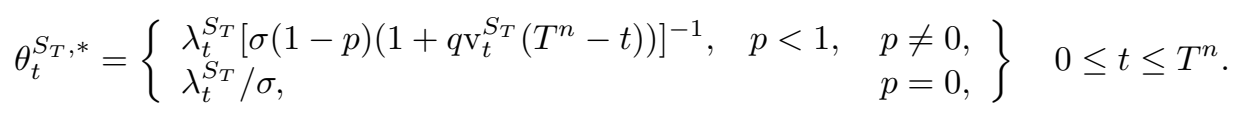

To conclude that the value function of the insider is as claimed, consider the following strategy in $\mathcal{A}\left(T ; \mathbb{F}^{S}\right)$. Follow the optimal strategy of the auxiliary problem until time $T^{n}$, then transfer all wealth from the risky asset to the risk-free one. Denote this strategy by $\theta^{S_{T}, n}$, given by

$$
\theta_{t}^{S_{T}, n}:=\mathbb{1}_{\left\{t \leq T^{n}\right\}} \theta^{S_{T}, *}, \quad 0 \leq t \leq T .
$$

Since this strategy is admissible for the original problem over the interval $[0, T]$, we have that

$$
u_{S_{T}}\left(x ; T^{n}, \mathbb{F}^{S_{T}}\right) \leq u_{S_{T}}\left(x ; T, \mathbb{F}^{S_{T}}\right) .
$$

Therefore, taking limits in (101) yields

$$
u_{S_{T}}\left(x ; T, \mathbb{F}^{S_{T}}\right) \geq \lim _{n \rightarrow+\infty} u_{S_{T}}\left(x ; T^{n}, \mathbb{F}^{S_{T}}\right)= \begin{cases}\infty, & p \in[0,1), \\ 0, & p<0,\end{cases}
$$

Since $u_{S_{T}}(x) \leq+\infty$ for $p \in(0,1)$ and $u_{S_{T}}(x) \leq 0$ for $p \in(-\infty, 0)$, the result follows.

\section{Conclusion}

We have studied the effect of insider information on the estimation of an unknown stock price drift and on the optimal strategy to maximise expected utility of wealth, in a log-Brownian setting. We showed that filtering with additional information leaves the Kalman-Bucy equations intact, with the exception of modified initial conditions. Applying this filtering algorithm to the scenario where an insider estimates the unknown drift parameter based on his anticipative information (either Brownian or stock price-based) and stock return observations, we found that the insider has an advantage over the regular trader in all cases. Anticipative stock price information is superior to anticipative Brownian information in the sense that the variance of the estimate of the unknown drift is lower. This ultimately leads to an optimal trading strategy that is generally more aggressive in terms of stock holdings. In the case where the insider possesses precise knowledge of the future stock price, the maximal expected utility blows up, This is to be compared with the classical scenario considered in Pikovsky and Karatzas [27], in which the stock price drift is known with certainty, and in which precise information of any kind leads to unbounded utility.

\section{References}

[1] K. K. Aase, T. BuUland, And B. ØKSEndal, Strategic insider trading equilibrium: a forward integration approach. Preprint, 2007.

[2] J. Amendinger, D. Becherer, And M. Schweizer, A monetary value for initial information in portfolio optimization, Finance Stoch., 7 (2003), pp. 29-46.

[3] J. Amendinger, P. Imkeller, And M. Schweizer, Additional logarithmic utility of an insider, Stochastic Process. Appl., 75 (1998), pp. 263-286.

[4] S. Ankirchner, S. Dereich, And P. Imkeller, The Shannon information of filtrations and the additional logarithmic utility of insiders, Ann. Probab., 34 (2006), pp. 743-778.

[5] K. BACK, Insider trading in continuous time, Rev. Fin. Studies, 5 (1992), pp. 387-409.

[6] F. Baudoin And L. NGuYen-NGoc, The financial value of a weak information on a financial market, Finance Stoch., 8 (2004), pp. 415-435.

[7] T. BJÖRK, M. H. A. DAVIS, AND C. LANDÉN, Optimal investment under partial information. Preprint, 2008. 
[8] S. Brendle, Portfolio selection under incomplete information, Stochastic Process. Appl., 116 (2006), pp. 701-723.

[9] L. CAmpI, Some results on quadratic hedging with insider trading, Stochastics, 77 (2005), pp. 327348.

[10] L. CAmpi AND U. ÇETIN, Insider trading in an equilibrium model with default: a passage from reduced-form to structural modelling, Finance Stoch., 11 (2007), pp. 591-602.

[11] K.-H. CHo, Continuous auctions and insider trading: uniqueness and risk aversion, Finance Stoch., 7 (2003), pp. $47-71$.

[12] J. M. Corcuera, P. Imkeller, A. Kohatsu-Higa, and D. Nualart, Additional utility of insiders with imperfect dynamical information, Finance Stoch., 8 (2004), pp. 437-450.

[13] A. Danilova, Stock market insider trading in continuous time with imperfect dynamic information. Stochastics, forthcoming, 2010.

[14] W. H. Fleming And R. W. Rishel, Deterministic and stochastic optimal control, Springer-Verlag, Berlin, 1975. Applications of Mathematics, No. 1.

[15] C. Hillairet, Comparison of insiders' optimal strategies depending on the type of side-information, Stochastic Process. Appl., 115 (2005), pp. 1603-1627.

[16] P. Imkeller, Random times at which insiders can have free lunches, Stoch. Stoch. Rep., 74 (2002), pp. $465-487$.

[17] _ Malliavin's calculus in insider models: additional utility and free lunches, Math. Finance, 13 (2003), pp. 153-169. Conference on Applications of Malliavin Calculus in Finance (Rocquencourt, 2001).

[18] J. JACOD, Grossissement initial, hypotheèse (H') et théorème de Girsanov, in Grossissements de filtrations: exemples et applications, T. Jeulin and M. Yor, eds., vol. 1118 of Lecture Notes in Mathematics, Springer-Verlag, Berlin, 1985, pp. 15-35.

[19] I. KARATzAS, Lectures on the mathematics of finance, vol. 8 of CRM Monograph Series, American Mathematical Society, Providence, RI, 1997.

[20] A. Kohatsu-Higa And A. Sulem, Utility maximization in an insider influenced market, Math. Finance, 16 (2006), pp. 153-179.

[21] A. Kyle, Continuous auctions and insider trading, Econometrics, 53 (1985), pp. 1315-1336.

[22] P. LAKNER, Optimal trading strategy for an investor: the case of partial information, Stochastic Process. Appl., 76 (1998), pp. 77-97.

[23] R. S. Liptser and A. N. Shiryaev, Statistics of random processes. I: General theory, SpringerVerlag, Berlin, second ed., 2001.

[24] R. Mansuy And M. Yor, Random times and enlargements of filtrations in a Brownian setting, vol. 1873 of Lecture Notes in Mathematics, Springer-Verlag, Berlin, 2006.

[25] M. Monoyios, Utility-based valuation and hedging of basis risk with partial information. Submitted, 2009.

[26] H. Pham And M.-C. Quenez, Optimal portfolio in partially observed stochastic volatility models, Ann. Appl. Probab., 11 (2001), pp. 210-238.

[27] I. Pikovsky and I. Karatzas, Anticipative portfolio optimization, Adv. in Appl. Probab., 28 (1996), pp. 1095-1122.

[28] L. C. G. Rogers, The relaxed investor and parameter uncertainty, Finance Stoch., 5 (2001), pp. 131-154.

[29] J. XIOng AND X. Y. ZHOU, Mean-variance portfolio selection under partial information, SIAM J. Control Optim., 46 (2007), pp. 156-175.

[30] M. YoR, Some aspects of Brownian motion. Part II, Lectures in Mathematics ETH Zürich, Birkhäuser Verlag, Basel, 1997. Some recent martingale problems. 